JOURNAL OF

SYMPLECTIC GEOMETRY

Volume 4, Number 3, 345-372, 2007

\title{
THE FUNDAMENTAL GROUP OF SYMPLECTIC MANIFOLDS WITH HAMILTONIAN LIE GROUP ACTIONS
}

\author{
HUi LI
}

Let $(M, \omega)$ be a connected, compact symplectic manifold equipped with a Hamiltonian $G$ action, where $G$ is a connected compact Lie group. Let $\phi$ be the moment map. In [12], we proved the following result for $G=S^{1}$ action: as fundamental groups of topological spaces, $\pi_{1}(M) \cong \pi_{1}\left(M_{\text {red }}\right)$, where $M_{\text {red }}$ is the symplectic quotient at any value of the moment map $\phi$, and $\cong$ denotes "isomorphic to". In this paper, we generalize this result to other connected compact Lie group $G$ actions. We also prove that the above fundamental group is isomorphic to that of $M / G$. We briefly discuss the generalization of the first part of the results to non-compact manifolds with proper moment maps.

\section{Introduction}

Let $(M, \omega)$ be a connected, compact symplectic manifold. Let us assume a connected compact Lie group $G$ acts on $M$ in a Hamiltonian fashion with moment map $\phi: M \rightarrow \mathfrak{g}^{*}$, where $\mathfrak{g}^{*}$ is the dual Lie algebra of $G$. Assume $\phi$ is equivariant with respect to the $G$ action, where $G$ acts on $\mathfrak{g}^{*}$ by the co-adjoint action. Take a moment map value $a$ in $\mathfrak{g}^{*}$, the space $M_{G \cdot a}=$ $\phi^{-1}(G \cdot a) / G$ is called the symplectic quotient or the reduced space at the co-adjoint orbit $G \cdot a$. If $G_{a}$ is the stabilizer group of $a$ under the co-adjoint action, by equivariance of the moment map, the two reduced spaces are equal: $M_{a}=\phi^{-1}(a) / G_{a}=\phi^{-1}(G \cdot a) / G=M_{G \cdot a}$. We will use the two notations interchangeably. The space $M_{G \cdot a}$ can be a smooth symplectic manifold, or a symplectic orbifold, or a symplectic stratified space. The 
space $M_{G \cdot a}$, as a topological space, has a well defined fundamental group. One has the notion of orbifold fundamental group which is different (see [4] or [14] for the definition of orbifold $\pi_{1}$, and see the example following Theorem 1.1). In [12], we proved the following theorem:

Theorem 1.1. Let $(M, \omega)$ be a connected, compact symplectic manifold equipped with a Hamiltonian $S^{1}$ action. Then, as fundamental groups of topological spaces, $\pi_{1}(M) \cong \pi_{1}($ minimum $) \cong \pi_{1}($ maximum $) \cong \pi_{1}\left(M_{\text {red }}\right)$, where $M_{\text {red }}$ is the symplectic quotient at any value in the image of the moment map $\phi$.

The proof mainly relies on Morse-Bott theory and symplectic reduction. It closely links the fundamental group of the "manifold under a value $a$ " $M^{a}=\{x \in M \mid \phi(x) \leq a\}$ with the fundamental group of the reduced spaces at values equal to or lower than $a$.

The above theorem is not true for "orbifold fundamental group". For example, let $S^{1}$ act on $\left(S^{2} \times S^{2}, 2 \rho \oplus \rho\right)$ (where $\rho$ is the standard symplectic form on $\left.S^{2}\right)$ by $\lambda\left(z_{1}, z_{2}\right)=\left(\lambda^{2} z_{1}, \lambda z_{2}\right)$. Let 0 be the minimal value of the moment map. Then for $a \in(1,2), M_{a}$ is an orbifold which is homeomorphic to $S^{2}$ and has two $\mathbb{Z}_{2}$ singularities. The orbifold $\pi_{1}$ of $M_{a}$ is $\mathbb{Z}_{2}$, but the $\pi_{1}$ of $M_{a}$ as a topological space is trivial.

Theorem 1.1 is not true for non-compact symplectic manifolds. For instance, take $S^{1} \times \mathbb{R}$ and let $S^{1}$ act by rotating the first factor. This action is Hamiltonian with moment map being the projection to $\mathbb{R}$. We see that each reduced space is a point.

In this paper, we generalize Theorem 1.1 to the case of torus actions and to the case of non-abelian group actions. We state this generalization in two theorems, separating the abelian and non-abelian group actions.

Theorem 1.2. Let $(M, \omega)$ be a connected, compact symplectic manifold equipped with a Hamiltonian torus $T^{n}$ action $(n>1)$ with moment map $\phi$. Then, as fundamental groups of topological spaces, $\pi_{1}(M) \cong$ $\pi_{1}\left(M_{\text {red }}\right)$, where $M_{\text {red }}$ is the symplectic quotient at any value of the moment map $\phi$.

Theorem 1.3. Let $(M, \omega)$ be a connected, compact symplectic manifold equipped with a Hamiltonian $G$ action with moment map $\phi$, where $G$ is a connected compact non-abelian Lie group. Then, as fundamental groups of topological spaces, $\pi_{1}(M) \cong \pi_{1}\left(M_{\text {red }}\right)$, where $M_{\text {red }}$ is the symplectic quotient at any co-adjoint orbit in the image of the moment map $\phi$.

In order to explain the main ideas of the proofs, let us recall the following convexity theorems.

Theorem 1.4. ([2] or $[\mathbf{6}])$ Let $(M, \omega)$ be a connected compact symplectic manifold equipped with a Hamiltonian torus $T$ action. Let $\phi$ be the 
moment map. Then

1. For each value $a \in i m(\phi), \phi^{-1}(a)$ is connected.

2. The moment map image $\phi(M)$ is a convex polytope $\triangle$. It is the convex hull of the images of the fixed point sets of $T$.

Theorem 1.5. ([9] or $[\mathbf{1 1}])$ Let $(M, \omega)$ be a connected, compact symplectic manifold equipped with a Hamiltonian $G$ action with moment map $\phi$, where $G$ is a connected compact non-abelian Lie group. Let $\mathbf{t}_{+}^{*}$ be a fixed closed positive Weyl chamber of $\mathfrak{g}^{*}$. Then

1. For each co-adjoint orbit $\mathcal{O}$ in the image of $\phi, \phi^{-1}(\mathcal{O})$ is connected.

2. The image $\phi(M) \cap \mathfrak{t}_{+}^{*}=\triangle^{\prime}$ is a convex polytope.

The proofs of Theorem 1.2 and Theorem 1.3 mainly consist two steps. Step 1, we use Theorem 1.1 and "maximal" value on the moment polytope to prove that the reduced space at this value has the fundamental group of $M$. Step 2, we prove that all the reduced spaces have isomorphic fundamental groups. Step 1 is not hard. For Step 2, let us mainly explain the idea for the case of an abelian group $G=T$ action. The moment polytope $\triangle$ in Theorem 1.4 consists of faces with different dimensions. We call the values in the maximal dimensional faces generic values. We call the values on other faces singular values. For two generic values near each other, by the equivariant coisotropic embedding theorem, we show that the reduced spaces at these two values are diffeomorphic. To compare the fundamental groups of the reduced spaces at a singular value $c$ and at a nearby generic value $a$, we mainly use two facts. The first fact is, the gradient flow of the moment map square gives an equivariant deformation retraction from $\phi^{-1}(U)$ to $\phi^{-1}(c)$, where $U$ is a small open neighborhood of $c$ (see [15] or $[\mathbf{1 0}])$. Therefore, $\pi_{1}\left(\phi^{-1}(U) / T\right) \cong \pi_{1}\left(\phi^{-1}(c) / T\right) \cong \pi_{1}\left(M_{c}\right)$. The second fact is the key step toward solving the problem. It is a removing lemma. The space $\phi^{-1}(U)$ is a $T$-invariant smooth manifold. The quotient space $\phi^{-1}(U) / T$ is a stratified space. We will remove in a certain order the singular strata and, possibly, some piece of the generic stratum when necessary from this quotient. We prove that, each time we remove, the resulting space has isomorphic fundamental group as that of $\phi^{-1}(U) / T$. We do the removing until we get a space which has the homotopy type of $M_{a}$. This proves that $\pi_{1}\left(M_{c}\right) \cong \pi_{1}\left(M_{a}\right)$. For convenience, in the above argument, we may take a subset $\bar{U}^{\prime}$ of $U$ and prove the above fact by using $\bar{U}^{\prime}$ (see Lemma 3.8). For the case of a non-abelian Lie group $G$ action, the closed positive Weyl chamber consists of faces with different dimensions each of which has a different stabilizer group under the co-adjoint action. We use the symplectic cross-section theorem (see Theorem 4.10) to reduce the proof of Step 2 for the values on the maximal dimensional face of $\mathfrak{t}_{+}^{*}$ (which contains values of $\phi)$ to a torus action case. For moment map values on other faces of $\mathfrak{t}_{+}^{*}$, we 
use the symplectic cross-section theorem and a similar idea as in the case of an abelian group action.

Remark 1.1. By the above description (and by the method of the proof of Theorem 1.1 in [12]), the isomorphisms of the fundamental groups between some of the two spaces are obtained by the fact that the two spaces are diffeomorphic, homotopy equivalent, or by using the Van-Kampen theorem when we do removing (or gluing). When we do removing from a space, we may need to do it multiple times. Each time we remove, we prove that the resulting space has isomorphic fundamental group as the previous one. Each time we use the Van-Kampen theorem, the base point is naturally taken in the connected intersection of the two connected open sets which cover the space. For the above reasons, in this paper, to simplify notation, when the context is clear, we will omit writing base point when we write $\pi_{1}$ of a space. The isomorphisms of the fundamental groups between the rest of the two spaces are obtained by transitivity.

The method of the proofs of Theorem 1.2 and Theorem 1.3 for the part that all the reduced spaces have isomorphic fundamental groups can be applied to the case of non-compact manifolds with proper moment maps. Regarding the fundamental group of the manifold $M$, we have the following observation. If the moment map $\phi$ has no critical values, then $\phi$ is a proper submersion from $M$ to $\mathfrak{g}^{*}$. By Ehresmann's Lemma, $\phi$ gives a fibration from $M$ to $\mathfrak{g}^{*}$ with connected fiber ([9] or [11]) diffeomorphic to $\phi^{-1}(a)$ for some $a \in i m(\phi)$. By the homotopy exact sequence for fibrations, we have $\pi_{1}(M) \cong \pi_{1}\left(\phi^{-1}(a)\right.$ ) (see the example of $S^{1} \times \mathbb{R}$ following Theorem 1.1). This may not be the fundamental group of the reduced spaces. If the moment map $\phi$ has critical values, one may still be able to prove that $\pi_{1}(M) \cong \pi_{1}\left(M_{\text {red }}\right)$. There can be different approaches for this.

Another interesting fact that the method implies is:

Theorem 1.6. Let $(M, \omega)$ be a connected, compact symplectic manifold equipped with a Hamiltonian $G$ action with moment map $\phi$, where $G$ is a connected compact Lie group. Then, as fundamental groups of topological spaces, $\pi_{1}(M / G) \cong \pi_{1}\left(M_{r e d}\right)$, where $M_{\text {red }}$ is the symplectic quotient at any co-adjoint orbit in the image of the moment map $\phi$.

In this paper, when we say fundamental group, we mean the fundamental group of the topological space, without explicitly saying so.

A brief organization of the paper. In Section 2, we will recall facts about proper compact Lie group actions and stratified spaces. Most importantly, we build blocks on removing certain strata from a stratified space which will keep $\pi_{1}$ of the resulting space the same as that of the original space. In 
Section 3, we prove Theorem 1.2. In Section 4, we recall the symplectic crosssection theorem and the local normal form theorem. These are important tools for studying non-abelian Hamiltonian Lie group actions. In Section 5, we prove Theorem 1.3 for rank 1 connected compact non-abelian Lie group actions. This not only gives us an example of Theorem 1.3, but also allows us to see the method of proof for general non-abelian connected compact Lie group actions. In Section 6, we prove Theorem 1.3. In Section 7, we prove Theorem 1.6.

\section{Lie group actions and stratified spaces}

In this section, we will recall the definition of a stratified space. For proper Lie group actions on a smooth manifold where slice theorem applies, Bredon has shown that the quotient space is a stratified space. We will recall this proof, and emphasize important points about stratified spaces. Then, we state two lemmas and Armstrong's theorem which are very useful in the subsequent sections about removing strata from a stratified space.

One may refer to $[\mathbf{1 3}]$ for the following definition of stratified spaces.

Definition 2.1. Let $X$ be a Hausdorff and paracompact topological space and let $\mathcal{J}$ be a partially ordered set with order relation denoted by $\leq$. A $\mathcal{J}$-decomposition of $X$ is a locally finite collection of disjoint, locally closed manifolds $S_{i} \subset X$ (one for each $i \in \mathcal{J}$ ) called pieces such that

(i) $X=\cup_{i \in \mathcal{J}} S_{i}$;

(ii) $S_{i} \cap \bar{S}_{j} \neq \emptyset \Leftrightarrow S_{i} \subset \bar{S}_{j} \Leftrightarrow i \leq j$.

We call the space $X$ a $\mathcal{J}$-decomposed space.

Definition 2.2. A decomposed space $X$ is called a stratified space if the pieces of $X$, called strata, satisfy the following condition:

Given a point $x$ in a piece $S$, there exist an open neighborhood $\tilde{U}$ of $x$ in $X$, an open ball $B$ around $x$ in $S$, a compact stratified space $L$, called the link of $\mathrm{x}$, and a homeomorphism

$$
\varphi: B \times \stackrel{\circ}{C} L \longrightarrow \tilde{U}
$$

that preserves the decompositions.

In the above definition, $\stackrel{\circ}{C} L$ is the space obtained by collapsing the boundary $L \times 0$ of the half-open cylinder $L \times[0, \infty)$ to a point.

From now on, for convenience, we may not specify the point $x$ in a (connected) stratum $S$, and we will call the link of $\mathbf{x}$ the link of $\mathbf{S}$.

Let us recall Bredon's idea (see [3]) about the fact that the quotient space of a proper Lie group action on a smooth manifold is a stratified space. Assume a compact Lie group $G$ acts smoothly on a smooth manifold $N$. Assume that we have chosen a $G$-invariant metric on $N$. By the 
slice theorem, a neighborhood in $N$ of an orbit with isotropy type $(H)$ (subgroups conjugate to $H$ ) is isomorphic to $G \times_{H} D$, where $D$ is a disk in the orthogonal complement of the tangent space to the orbit on which $H$ acts linearly. The action of $H$ on $D$ is equivalent to an "orthogonal" action, i.e., $H$ acts as a subgroup of $O(n)$, where $n=\operatorname{dim} D$ (See Theorem 0.3.5 in $[3])$. So a neighborhood of the point $G / H$ in $N / G$ is isomorphic to $D / H$. Let $D=D_{1} \times D_{2}$, where $D_{1}$ is the disk consisting of fixed vectors by $H$. So the neighborhood of $G / H$ in $N / G$ is isomorphic to $D_{1} \times \stackrel{\circ}{C} L$, where $L=S\left(D_{2}\right) / H$ with $S\left(D_{2}\right)$ being a "sphere" of $D_{2}$. It is clear that the link $L$ is connected if the dimension of $D_{2}$ is bigger than 1 , and the orbit types in $L$ have isotropy groups no bigger than $H$. So a small neighborhood in $N / G$ of a point in the $(H)$-stratum consists of $\left(K_{i}\right)$-strata, where $K_{i}$ is conjugate to a subgroup of $H$. In other words, the $(H)$-stratum can only be contained in the closure of $(K)$-strata, where $K$ is sub-conjugate to $H$. If we take a connected component $S$ of the $(H)$-stratum in $N / G$, then a neighborhood of $S$ in $N / G$ can be seen as the total space of a fiber bundle over $S$ with fiber $\stackrel{\circ}{C} L$.

In Sections 3, 5, and 6, we will compute links of points. For convenience, we summarize the above computation of a link in the following Lemma.

Lemma 2.1. Let a compact connected Lie group G act smoothly on a smooth connected manifold $N$. Then the quotient space $N / G$ is a stratified space. Let $S$ be a connected component of a stratum of $N / G$ with isotropy group conjugate to $H$. Let $G \times{ }_{H} D$ be a neighborhood in $N$ of an orbit with isotropy type $(H)((H)$ denotes the collection of subgroups conjugate to $H)$. Let $D=D_{1} \times D_{2}$, where $D_{1}$ is the disk consisting of fixed vectors by $H$. Let $S\left(D_{2}\right)$ be a sphere in $D_{2}$. Then the link of the corresponding point in $S$ (or the link of $S$ ) is $L_{H}=S\left(D_{2}\right) / H$. If $\operatorname{dim}\left(D_{2}\right)>1$, then $L_{H}$ is connected.

We will mainly use the following lemma and theorem to determine whether the link of a connected component of a stratum is simply connected.

Lemma 2.2. (Corollary 6.3 in [3]) If $X$ is an arcwise connected G-space, $G$ compact Lie, and if there is an orbit which is connected, then the fundamental group of $X$ maps onto that of $X / G$.

Theorem 2.7. ([1] ) Let $G$ be a compact Lie group acting on a connected, locally path connected, simply connected, locally compact metric space $X$. Let $H$ be the smallest normal subgroup of $G$ which contains the identity component of $G$ and all those elements of $G$ which have fixed points. Then the fundamental group of the orbit space $X / G$ is isomorphic to $G / H$.

Note that, if $G$ is connected, the above theorem claims the same as Lemma 2.2 for simply connected $X$. 
The next lemma is the key ingredient for claiming that the fundamental group remains unchanged after removing a stratum.

Lemma 2.3. Let a compact connected Lie group G act smoothly on a smooth connected manifold $N$ such that $N / G$ is a stratified space. Let $S$ be a connected component of a stratum in $N / G$ such that no other strata are contained in the closure of $S$. Assume the link $L_{S}$ of $S$ is connected and simply connected. Then $\pi_{1}(N / G) \cong \pi_{1}(N / G-S)$.

Proof. Let $O_{1}$ be an open neighborhood of $S$ in $N / G$ such that $O_{1}$ fibers over $S$ with fiber $\stackrel{\circ}{C} L_{S}$. Since $\stackrel{\circ}{C} L_{S}$ is simply connected, we have $\pi_{1}\left(O_{1}\right) \cong \pi_{1}(S)$.

Take $O_{2}=N / G-S$. Then $O_{1} \cap O_{2}$ fibers over $S$ with fiber $\stackrel{\circ}{C} L_{S}-0$ which is homotopy equivalent to $L_{S}$. Since $L_{S}$ is connected and simply connected by assumption, $\pi_{1}\left(O_{1} \cap O_{2}\right) \cong \pi_{1}(S)$. By the Van-Kampen theorem, $\pi_{1}(N / G) \cong \pi_{1}\left(O_{1}\right) *_{\pi_{1}\left(O_{1} \cap O_{2}\right)} \pi_{1}\left(O_{2}\right) \cong \pi_{1}(N / G-S)$.

\section{Proof of Theorem $\mathbf{1 . 2}$}

By Theorem 1.4, under the assumptions of Theorem 1.2, the moment map image is a convex polytope.

Now, let us recall the following well known facts about connected compact Lie group actions. One may refer to [6] and the references cited in [6].

Proposition 3.1. Let $X$ be a connected manifold and $G$ be a connected compact Lie group acting smoothly on $X$. Let $G_{x}$ be the stabilizer group of $x$. Let $r=\min \operatorname{dim} G_{x}$. Let $X_{i}$ be the set of $x$ 's for which $\operatorname{dim} G_{x} \geq i+r$. Then

1. If $X$ is compact, up to conjugacy, only a finite number of subgroups of $G$ occur as stabilizer groups of points of $X$. The subset $X_{i}$ has codimension $\geq i+1($ for $i=1,2, \ldots)$ in $X$.

2. Let $H$ be a closed subgroup of $G$. Let $X_{H}=\left\{x \in X \mid G_{x}=H\right\}$. Then $X_{H}$ is a submanifold of $X$. If $X$ is a symplectic manifold and $G$ acts symplecticly, then $X_{H}$ is a symplectic submanifold of $X$.

From 1. of the above proposition, $X_{r}$ is open dense and connected. Up to conjugacy, let us call the stabilizer type of the points in $X_{r}$ principal stabilizer type.

Now we come back to our symplectic manifold $(M, \omega)$ with a Hamiltonian $G$ action, where $G$ is a connected compact Lie group. By definition of the moment map $\phi$, for each $X \in \mathfrak{g}$, we have $i_{X_{M}} \omega=d\langle\phi, X\rangle$, where $X_{M}$ is the vector field on $M$ generated by $X$. From this, we easily derive

Lemma 3.4. Let $m \in M$. Let $G_{m}$ be the stabilizer group of $m$ in $G$ and let $\mathfrak{g}_{m}$ be its Lie algebra. Then the image of $d \phi_{m}: T_{m} \rightarrow \mathfrak{g}^{*}$ is the annihilator in $\mathfrak{g}^{*}$ of $\mathfrak{g}_{m}$. 
For the manifold $M$ in Theorem 1.4, more explicitly, it can be stratified according to the isotropy groups. Let $T_{1}, T_{2}, \ldots, T_{N}$ be the subgroups of $T$ which occur as stabilizer groups of points of $M$. Let $M_{i}$ be the set of points in $M$ for which the stabilizer group is $T_{i}$. By relabeling, we may assume that the $M_{i}$ 's are connected (So some $T_{i}$ ' may be repeated). Then $M$ is a disjoint union:

$$
M=\bigcup_{i=1}^{N} M_{i} .
$$

The moment map images of the fixed point set components of $T$ are called the vertices of $\phi$. The vertices of $\phi$ can be "real" vertices on the boundary of $\triangle$, and can be "vertices" inside $\triangle$.

Each $M_{i}$ is a $T$-invariant symplectic submanifold of $M, \phi\left(M_{i}\right)$ is an open subset of the affine plane $a_{i}+\mathfrak{t}_{i}^{\perp}$, where $a_{i}$ is a vector in $\mathfrak{t}^{*}, \mathfrak{t}_{i}=\operatorname{Lie}\left(T_{i}\right)$ and $\mathfrak{t}_{i}^{\perp}$ is the annihilator (or perpendicular) of $\mathfrak{t}_{i}$ in $\mathfrak{t}^{*}$ (or in $\mathfrak{t}$ ). Moreover, $\phi\left(M_{i}\right)$ is the union of a finite number of convex sets each of which is the convex hull of a collection of the vertices.

By the above description, $\triangle$ consists of faces with different dimensions.

Remark 3.2. We may assume that $\triangle$ contains an open subset of $\mathfrak{t}^{*}$. By the definition of the moment map, this is the same as assuming that the $T$ action has finite generic stabilizer group. Moreover, we assume that $\bigcap_{m \in M} T_{m}=1$, where $T_{m}$ is the stabilizer group of $m$. If this is not the case, we divide $T$ by the common stabilizer group and consider the quotient torus action. Let us call the values of $\phi$ in the open set regular values. Clearly, the set of regular values is open and dense in $i m(\phi)$.

Let us call a connected set of regular values of $\phi$ a (connected) chamber of $\triangle$. The moment map image $\triangle$ may have one or more than one connected chambers.

Remark 3.3. Note that the $M_{i}$ 's are disjoint, but the $\phi\left(M_{i}\right)$ 's may not be disjoint (they are disjoint if $(M, \omega, T, \phi)$ is a completely integrable system.). For instance, regular points on $M$, i.e., points with finite stabilizer groups can be mapped to non-open faces. One may see this easily for $S^{1}$ actions, and then generalize to $T$ actions. For a boundary vertex $v, \phi^{-1}(v)$ only consists of one fixed point set component; for a vertex inside $\triangle$, this cannot be true. Indeed, by the following Theorem 3.9, a neighborhood in $M$ of a fixed point $x$ is isomorphic to a $T$ representation $V=W \oplus V^{T}$, and $\left.\phi\right|_{V}$ is the moment map for the $T$ action on $W$. If $\phi(x)$ is on the boundary of $\triangle$, then at least one subcircle of $T$ acts on $W$ with weights all positive, so $\phi^{-1}(0)$ only consists of the fixed point set component containing $x$. One may see similarly that for an interior vertex $v, \phi^{-1}(v)$ contains more points than the fixed point set itself. For a wall $\mathcal{W}$ on the boundary, $\phi^{-1}(\mathcal{W})$ is fixed by the circle generated by the direction perpendicular to $\mathcal{W}$. For a 
wall $\mathcal{W}$ inside $\triangle, \phi^{-1}(\mathcal{W})$ consists more than just a submanifold which is fixed by the circle generated by the direction perpendicular to $\mathcal{W}$.

Lemma 3.5. Suppose $X_{1}, X_{2}, \ldots, X_{k}$ are connected components of the fixed point set of $T$ such that $\phi\left(X_{i}\right), i=1, \ldots, k$ are boundary vertices $v_{i}, i=$ $1, \ldots, k$ of the polytope $\triangle$. Then $\pi_{1}\left(M_{v_{i}}\right) \cong \pi_{1}\left(X_{i}\right) \cong \pi_{1}(M)$, for $i=1, \ldots, k$.

Proof. The first equality is clear since $\phi^{-1}\left(v_{i}\right)=X_{i}$ (see Remark 3.3) and $X_{i}$ is fixed by $T$. To prove the second equality, choose a sub-circle in $T$ such that $v_{i}$ is the maximal value of the moment map for the circle action. Then we apply Theorem 1.1.

Remark 3.4. Although we are proving Theorem 1.2, the following proofs of Lemmas 3.6 and 3.7 cover the case of $S^{1}$ actions.

Lemma 3.6. For two values $a, b$ near each other in one connected chamber of $\triangle$, we have $\pi_{1}\left(M_{a}\right) \cong \pi_{1}\left(M_{b}\right)$. Therefore, by connectivity of the chamber, for all values $a$ in this chamber, $\pi_{1}\left(M_{a}\right)^{\prime} s$ are all isomorphic.

Proof. Take two regular values $a$ and $b$ close enough. By the equivariant coisotropy embedding theorem, there exists a small neighborhood $U$ containing $a$ and $b$ such that $U$ consists of regular values, and $\phi^{-1}(U)$ is isomorphic to $\phi^{-1}(a) \times U$, where $T$ acts on $\phi^{-1}(a)$ and the moment map is the projection to $U$. So $M_{a}$ is diffeomorphic to $M_{b}$. So $\pi_{1}\left(M_{a}\right) \cong \pi_{1}\left(M_{b}\right)$.

Lemma 3.7. Let $c$ be a non-regular value on $\triangle$. Let a be a regular value such that it is very near $c$. Then $\pi_{1}\left(M_{c}\right) \cong \pi_{1}\left(M_{a}\right)$.

Proof of Theorem 1.2:

Proof. Theorem 1.2 follows from Lemmas 3.5, 3.6, and 3.7.

Lemma 3.7 follows from the following Lemma 3.8. Let us first recall the following theorem on the convergence of the gradient flow of the moment map square:

Theorem 3.8. ([15] or $[\mathbf{1 0}])$ Let $(M, \omega)$ be a connected Hamiltonian Gmanifold with proper moment map $\phi$, where $G$ is a connected compact Lie group. Choose a G-invariant metric on $M$. Assume that the moment map image intersects a neighborhood of 0 (the image not necessarily fills an open neighborhood of 0$)$. Then there exists a $G$-invariant open neighborhood $U \subset \mathfrak{g}^{*}$ of 0 such that the negative gradient flow of the moment map square induces a $G$-equivariant deformation retraction from $\phi^{-1}(U)$ to $\phi^{-1}(0)$.

If $G$ is a torus, we can always shift the moment map (by a constant) such that a value $c$ we consider corresponds to the 0 value of the new moment map, so without loss of generality, we can regard $c$ as 0 . 
Assume $G$ is a torus. Take $U$ as in Theorem 3.8. Let $U^{\prime}$ be the intersection of $U$ with a connected open chamber, and, let $\bar{U}^{\prime}$ be its closure in $U$. Then $\phi^{-1}\left(\bar{U}^{\prime}\right)$ also $G$-equivariantly deformation retracts to $\phi^{-1}(0)$.

Lemma 3.8. Let $c$ be a value on a singular face of $\triangle$, and let a be a regular value very near $c$. Let $U$ be a small open neighborhood of $c$ on $\triangle$ containing a such that $\phi^{-1}(U)$ equivariantly deformation retracts to $\phi^{-1}(c)$. Let $U^{\prime}$ be the intersection of $U$ with the connected open chamber containing a, and let $\bar{U}^{\prime}$ be its closure in $U$. Let $B$ be the set of values in $\bar{U}^{\prime}$ but not in $U^{\prime}$. Then $\pi_{1}\left(\phi^{-1}\left(\bar{U}^{\prime}\right) / T\right) \cong \pi_{1}\left(\phi^{-1}\left(\bar{U}^{\prime}\right) / T-\phi^{-1}(B) / T\right)$, i.e., $\pi_{1}\left(M_{c}\right) \cong \pi_{1}\left(M_{a}\right)$.

We could use the set $U$ itself, and apply a removing and flowing (by using the gradient flow) process to achieve $\pi_{1}\left(M_{c}\right) \cong \pi_{1}\left(M_{a}\right)$. I found that I still would have to do the above removing in the end. So taking $\bar{U}^{\prime}$ is more convenient.

In order to prove the above lemma, let us recall the following Local Normal Form theorem for abelian Lie group actions.

Theorem 3.9. (Local normal form) $([8])$ Let $(M, \omega)$ be a symplectic manifold with a Hamiltonian torus $T$ action. Let $H$ be the isotropy subgroup of a point $p$ in $M$. Then a neighborhood in $M$ of the orbit through $p$ is equivariantly symplectomorphic to $T \times_{H}\left(\mathfrak{b}^{\perp} \times V\right)$, where $\mathfrak{b}^{\perp}$ is the annihilator of $\mathfrak{b}=\operatorname{Lie}(H)$ in $\mathfrak{t}^{*}$ on which $H$ acts by the co-adjoint action (trivial in this case), and $V$ is a complex vector space on which $H$ acts linearly and symplectically.

The equivalence relation on $T \times_{H}\left(\mathfrak{b}^{\perp} \times V\right)$ is given by $(t, a, v) \approx$ $\left(t h^{-1}, a, h \cdot v\right)$ for $h \in H$.

The $T$ action on this local model is $t_{1} \cdot[t, a, v]=\left[t_{1} t, a, v\right]$, and the moment map on this local model is $\phi([t, a, v])=\phi(p)+a+\psi(v)$, where $\psi(v)$ is the moment map for the $H$ action on $V$.

Remark 3.5. By Theorem 3.9, if an orbit has stabilizer $H$, then the nearby orbits of this orbit have stabilizers no bigger than $H$. So, when we remove a connected stratum which is more singular, we will not destroy the link of its nearby (less singular) strata. If a face $\mathcal{F}$ of $\triangle$ is in the closure of the face $\mathcal{F}^{\prime}$, then $\phi^{-1}(\mathcal{F})$ contains more singular (with bigger stabilizer groups) strata than the strata in $\phi^{-1}\left(\mathcal{F}^{\prime}\right)$. In Lemma 3.8 , if $\mathcal{F}$ is the face in $U$ containing the singular value $c$, then it is the most degenerate face in $U$.

The proof of Lemma 3.8 is a removing process. According to Lemma 1.3, the quotient space of a smooth manifold by a compact Lie group action is a stratified space, and certain removing of strata from the quotient keeps $\pi_{1}$ of the resulting space the same as $\pi_{1}$ of this quotient itself. The $\phi^{-1}\left(\bar{U}^{\prime}\right)$ we took is not a manifold. Nevertheless, the analysis of the neighborhoods allows us to perform the removing in $\phi^{-1}\left(\bar{U}^{\prime}\right) / T$. 
Proof. Assume that $\mathcal{F}$ is the singular face in $U$ containing $c$. Let $m \geq 0$ be its dimension. The face $\mathcal{F}$ is the most degenerate face in $U$ and in $\bar{U}^{\prime}$. According to Remark 3.5, we first remove $\phi^{-1}(\mathcal{F}) / T$.

Assume the dimension of the torus $T$ is $n$. Let us identify the Lie algebra $\mathfrak{t}$ of $T$ with its dual $\mathfrak{t}^{*}$. The $m$-dimensional linear subspace $L$ which contains $\mathcal{F}$ as an open set generates a subtorus $T^{m}$. If $\mathcal{F}$ is not a wall, the complementary linear subspace of $L$ in $\mathfrak{t}$ spanned by the set $S$ of other directions of one-dimensional faces of $\bar{U}^{\prime}$ generates a complementary subtorus $T^{n-m}$. If $\mathcal{F}$ is a wall, then we take the direction orthogonal to $L$ as a complementary direction, and we take the complementary subtorus of $T^{m}$ generated by this direction. So, we have chosen a splitting $T=T^{n-m} \times T^{m}$.

Notice that the most singular stratum in $\phi^{-1}(\mathcal{F})$ has stabilizer $T^{n-m} \times \Gamma$, where $\Gamma$ is a finite subgroup of $T^{m}$. The set $\phi^{-1}(\mathcal{F})$ may contain other strata with stabilizer(s) of the form $H=\left(T_{1} \times \Gamma^{\prime}\right) \times \Gamma$, where $T_{1}$ is a connected subgroup of $T^{n-m}$ generated by directions of some 1-dimensional faces of $\bar{U}^{\prime}, \Gamma^{\prime}$ is a finite subgroup of a complementary group $T_{2}$ of $T_{1}$ in $T^{n-m}$. (We may have a different finite group $\Gamma$ which is a subgroup of the previous $\Gamma$. But this will not affect the proof, so we use the same notation). Here, we chose $T_{2}$ to be the subgroup of $T^{n-m}$ generated by the rest of the directions in $S$ of 1-dimensional faces of $\bar{U}^{\prime}$ when $\mathcal{F}$ is not a wall, and, we chose $T_{2}$ to be trivial or to be the subgroup generated by the orthogonal direction of $L$ when $\mathcal{F}$ is a wall (remember that in this case $n-m$ was 1 ).

By Theorem 3.9, a neighborhood in $M$ of an orbit with isotropy type $H$ is isomorphic to $A=T \times{ }_{H}\left(\mathbb{R}^{l} \times \mathbb{R}^{m} \times V\right)$. The moment map on $A$ is $\phi=a+b+\psi(v)$, where $a \in \mathbb{R}^{l}, b \in \mathbb{R}^{m}$, and $\psi$ is the moment map of the $H$ action on $V$ (we assumed that " $c=0$ "). We split $V=W \times V^{H}$, where $W$ has no non-zero fixed vectors by $H$. Then $\psi$ is just $\left.\psi\right|_{W}$. So $A \cap \phi^{-1}\left(\bar{U}^{\prime}\right)=T \times_{H}\left(\left(\mathbb{R}^{m} \times V^{H}\right) \times\left(\left(\mathbb{R}^{+}\right)^{l} \times W \cap \psi^{-1}\left(\bar{U}^{\prime}\right)\right)\right)$ (strictly speaking, the above $\mathbb{R}^{m}$ should be a small open disk in $\mathbb{R}^{m}$ corresponding to $\mathcal{F} \cap \bar{U}^{\prime}$ ), where $\left(\mathbb{R}^{+}\right)^{l}$ are the non-negative real (half) lines pointing towards $\bar{U}^{\prime}$. The $H$-stratum in $A \cap \phi^{-1}\left(\bar{U}^{\prime}\right)$ which was mapped to $\mathcal{F}$ is $T \times{ }_{H}\left(\mathbb{R}^{m} \times V^{H}\right)$. The link $L_{H}$ of the corresponding quotient $H$-stratum in $\left(A \cap \phi^{-1}\left(\bar{U}^{\prime}\right)\right) / T$ is $S\left(\left(\mathbb{R}^{+}\right)^{l} \times W \cap \psi^{-1}\left(\bar{U}^{\prime}\right)\right) / H$. Here, $S\left(\left(\mathbb{R}^{+}\right)^{l} \times W \cap \psi^{-1}\left(\bar{U}^{\prime}\right)\right)$ is the intersection of $S\left(\mathbb{R}^{l} \times W\right)$ with $\left(\mathbb{R}^{+}\right)^{l} \times$ $W \cap \psi^{-1}\left(\bar{U}^{\prime}\right)$. Now, we consider all the possible cases of $H$ (will correspond to different $l$ ).

1. In the case of $l=0$ (corresponding to $H=T^{n-m} \times \Gamma$ ), since we assumed that the moment map value fills $U^{\prime}$, the moment map $\left.\psi\right|_{W}$ has to be non-trivial. By Remark 3.6 and Lemma 3.9 below, the link is connected and simply connected.

2. In the case of $l \neq 0$ and $W \neq 0$, by Remark 3.7 and Lemma 3.10 below, the link $L_{H}$ is connected and simply connected. 
3. In the case of $l \neq 0$ and $W=0$ (all the points in $A \cap \phi^{-1}\left(\bar{U}^{\prime}\right)$ have the same stabilizer group $H$ which has to be the generic stabilizer group), the link $L_{H}=S\left(\left(\mathbb{R}^{+}\right)^{l}\right) / H=S\left(\left(\mathbb{R}^{+}\right)^{l}\right)$ is connected and simply connected.

If there are more singular faces left in $\bar{U}^{\prime}$, we remove similarly as above.

Remark 3.6. For Case 1 in the proof of Lemma 3.8, we needed to consider the quotient $\left(S(W) \cap \psi^{-1}\left(\bar{U}^{\prime}\right)\right) /\left(T^{n-m} \times \Gamma\right)$, where $W$ is a complex $\left(T^{n-m} \times\right.$ $\Gamma)$-representation isomorphic to some $(\mathbb{C})^{n}$ (it splits into a product of $\mathbb{C}$ ) on which $T^{n-m} \times \Gamma$ acts as a subgroup of the maximal torus of $U(n)$. Due to how cyclic group acts on $(\mathbb{C})^{n}$, and due to the fact that a finite group action does not contribute to $\psi$, we can first divide $W$ by $\Gamma$, we get $W / \Gamma$ homeomorphic to $W$. The action of $T^{n-m}$ on $W / \Gamma$ corresponds to a "weight change" comparing to the action of $T^{n-m}$ on $W$. Therefore, we can restrict attention to the case of the following lemma.

Lemma 3.9. Let $\mathbb{C}^{n}$ be an effective $T^{k}$ symplectic representation, where $T^{k}$ is a connected torus, and $k \leq n$. Let $\psi$ be the moment map for the $T^{k}$ action. Let $U^{\prime} \subset\left(\mathfrak{t}^{k}\right)^{*}$ be an open connected chamber consisting of regular values of $\psi$, and let $\bar{U}^{\prime}$ be its closure. Let $S^{\prime}=S^{2 n-1} \cap \psi^{-1}\left(\bar{U}^{\prime}\right)$. Then, the quotient $S^{\prime} / T^{k}$ is always connected and simply connected.

Proof. When $k=n, T^{n}$ corresponds to the maximal torus of $U(n)$. It acts on $\mathbb{C}^{n}$ in the standard way with moment map $\psi=\left(\left|z_{1}\right|^{2},\left|z_{2}\right|^{2}, \ldots,\left|z_{n}\right|^{2}\right)$. In this case, $S^{\prime}=S^{2 n-1}$. So $S^{\prime} / T^{k}$ is either a point when $n=1$, or it is connected and simply connected by Lemma 2.2 .

Now assume that $k<n$. We may assume that $n>1$. Then $T^{k}$ acts on $\mathbb{C}^{n}$ as a subtorus of $T^{n}$. Its moment map $\psi$ is the projection of the above moment map to the dual Lie algebra of $T^{k}$, i.e., $\psi=\alpha_{1}\left|z_{1}\right|^{2}+\alpha_{2}\left|z_{2}\right|^{2}+\cdots+\alpha_{n}\left|z_{n}\right|^{2}$, where $\alpha_{i}, i=1, \ldots, n$ are weight vectors in $\left(\mathfrak{t}^{k}\right)^{*}$. Then $\bar{U}^{\prime}$ is formed by the cone with non-negative coefficients spanned by $p$ with $p \geq k$ number of vectors among $\alpha_{i}, i=1, \ldots, n$. Without loss of generality, we assume that they are the first $p$ vectors. Any $k$ number of linearly independent vectors among them generates $T^{k}$. Take $k$ number of linearly independent vectors, say the first $k$ vectors, among the $p$ number of vectors, and write each of $\alpha_{i}, i=p+1, \ldots, n$ as linear combinations of $\alpha_{i}, i=1, \ldots, k: \alpha_{i}=a_{i 1} \alpha_{1}+\cdots+a_{i k} \alpha_{k}$ for $i=p+1, \ldots, n$. Then $\psi=\left(\left|z_{1}\right|^{2}+\sum_{i \geq p+1} a_{i 1}\left|z_{i}\right|^{2}\right) \alpha_{1}+\cdots+\left(\left|z_{k}\right|^{2}+\sum_{i \geq p+1} a_{i k}\left|z_{i}\right|^{2}\right) \alpha_{k}+$ $\left|z_{k+1}\right|^{2} \alpha_{k+1}+\cdots+\left|z_{p}\right|^{2} \alpha_{p}=\sum_{i \leq p} f_{i} \alpha_{i}$. So $\psi^{-1}\left(\bar{U}^{\prime}\right)=\left\{z \in \mathbb{C}^{n}: f_{i}(z) \geq\right.$ $0, i=1, \ldots, p\}$. Since the action is linear and the moment map is homogeneous, we only need to prove that $\left(\left(\mathbb{C}^{n}-0\right) \cap \psi^{-1}\left(\bar{U}^{\prime}\right)\right) / T^{k}$ is connected and simply connected. Now consider $\left(\mathbb{C}^{n}-0\right) \cap \psi^{-1}\left(\bar{U}^{\prime}\right)$. Since 0 (very singular) is taken away, we can perturb the set a little bit using the gradient flow of 
some $f_{i}$ without changing the topology so that the above intersection has the same topology as a union of the sets $A_{i}=\left\{z \in \mathbb{C}^{n}: f_{i}>0, f_{J} \geq 0\right\}$ for some $i=1, \ldots, p$, where $J=\{1, \ldots, i-1, i+1, \ldots, p\}$. We move the terms with negative coefficients in each of $f_{i}$ to the right hand side of $f_{i} \geq 0$. For a fixed set $A_{i}$, we equivariantly deformation retract all the $z_{l} s^{\prime}$ (which occured in $f_{i}>0$ and in $f_{J} \geq 0$ ) with $l>p$ which are not on the left hand side of $f_{i}$ to 0 . Now, the possibilities are: (a) The right hand sides of all $f_{J}$ are 0 , then the inequalities $f_{J} \geq 0$ do not give any condition. The inequality $f_{i}>0$ (after deforming the right hand side to 0 ) represents a copy of $\mathbb{C}^{*}$ or a simply connected sphere. So $A_{i}$ is deformed into $\mathbb{C}^{*}$ or a simply connected sphere or a product of one of them with some copies of $\mathbb{C}$ represented by some coordinates on the left hand sides of $f_{J}$ but not on the left hand side of $f_{i}$. So $A_{i} / T^{k}$ is connected and simply connected. (b) The right hand sides of $f_{J}$ are not 0 . Then, we perform some algebraic operations between the inequalities, we have a new $f_{i}$. We equivariantly deform the coordinates which are not on the left hand side of the new $f_{i}$ to 0 again, and, we may need to repeat this process until we have case (a) with a different $f_{i}$ from the original one. The set $\left(\left(\mathbb{C}^{n}-0\right) \cap \psi^{-1}\left(\bar{U}^{\prime}\right)\right) / T^{k}$ is obtained by gluing the connected and simply connected $A_{i} / T^{k} \mathrm{~s}$. Now, we only need to see that the intersection of each two of $A_{i} s^{\prime}$ is connected. We may treat the intersections similarly as the above (now we have two strict inequalities), i.e., we use algebraic operations and we use deformations until we can deform all the right hand sides to 0 . Now we have two strict inequalities $f_{i}>0, f_{j}>0$ with (quadratic terms and) positive coefficients. The inequalities represented by $f_{J^{\prime}} \geq 0$ (where $J^{\prime}=\{1, \ldots, p\}-\{i, j\}$ ) with positive coefficients do not give conditions. So the coordinates on the left hand side of $f_{J^{\prime}} \geq 0$ but not on the left hand sides of $f_{i}>0, f_{j}>0$ are free. If there are no common coordinates in $f_{i}>0$ and $f_{j}>0$, we see that the intersection is a product of connected sets therefore connected. Otherwise, by writing the intersection as a union of products of $\mathbb{C}^{*} s^{\prime}$ with $\mathbb{C} s^{\prime}$ which are connected and which have connected intersections, we see that, the intersection of the two $A_{i} s$ are connected. The Van-Kampen theorem justifies the conclusion.

Remark 3.7. In the local model $A=T \times{ }_{H}\left(\mathbb{R}^{l} \times \mathbb{R}^{m} \times V\right)$ with $H=T_{1} \times$ $\Gamma^{\prime} \times \Gamma$ we considered in the proof of Lemma 3.8, if we divide $A$ by $T^{m}$, we get $A / T^{m}=T^{n-m} \times{ }_{\left(T_{1} \times \Gamma^{\prime}\right)}\left(\left(\mathbb{R}^{l} \times W / \Gamma\right) \times\left(\mathbb{R}^{m} \times V^{H}\right)\right)=\left(T^{n-m} \times_{\left(T_{1} \times \Gamma^{\prime}\right)}\right.$ $\left.\left(\mathbb{R}^{l} \times W / \Gamma\right)\right) \times\left(\mathbb{R}^{m} \times V^{H}\right)$. Now, for Case 2 in the proof of Lemma 3.8, we "forget" the component $\left(\mathbb{R}^{m} \times V^{H}\right.$ ) (it was mapped to the face $\mathcal{F}$ ) and we count the fact that $W / \Gamma$ is homeomorphic to $W$ and the fact that a finite group does not contribute to the moment map, we may restrict attention to the case of the following lemma. In the following lemma, the 
$U^{\prime}$ is the intersection of the $U^{\prime}$ in Lemma 3.8 and the moment map image on $A^{\prime}$.

Lemma 3.10. Let $T$ be a d-dimensional torus acting in a local model (see Theorem 3.9) $A^{\prime}=T \times_{\left(T_{1} \times \Gamma^{\prime}\right)}\left(\mathbb{R}^{l} \times W\right)$, where $T_{1}$ is a connected subgroup with dimension less than $d($ so $l \neq 0), \Gamma^{\prime}$ is a finite subgroup, and $W$ is a complex $T_{1} \times \Gamma^{\prime}$ representation with no non-zero fixed vectors. Let the moment map of the $T$ action on $A^{\prime}$ be $\phi([t, a, z])=a+\psi(z)$. Let $U^{\prime} \subset \mathfrak{t}^{*}$ be an open connected chamber consisting of regular values of $\phi$, and let $\bar{U}^{\prime}$ be its closure. Let $S^{\prime}=S\left(\left(\mathbb{R}^{+}\right)^{l} \times W \cap \psi^{-1}\left(\bar{U}^{\prime}\right)\right)$. Then, the quotient $S^{\prime} /\left(T_{1} \times \Gamma^{\prime}\right)$ is connected and simply connected.

Proof. We can see (as we did before) that $S^{\prime} /\left(T_{1} \times \Gamma^{\prime}\right)$ is the link of the quotient $\left(T_{1} \times \Gamma^{\prime}\right)$-stratum in $\left(A^{\prime} \cap \phi^{-1}\left(\bar{U}^{\prime}\right)\right) / T$.

The group $T_{1} \times \Gamma^{\prime}$ acts on $\left(\mathbb{R}^{+}\right)^{l}$ trivially; and, for a similar reason as we made in Remark 3.6 about cyclic group action on $W$ and about its trivial contribution to $\psi$, we can "ignore" $\Gamma^{\prime}$, and consider $S^{\prime} / T_{1}$. Assume $T_{1}$ is of dimension $k(k+l=d)$. We use the same notations as we did in the proof of Lemma 3.9, we have (assume $W$ is isomorphic to some $\mathbb{C}^{n}$ ) $W \cap \psi^{-1}\left(\bar{U}^{\prime}\right)=\left\{z \in \mathbb{C}^{n}: f_{i}(z) \geq 0, i=1, \ldots\right\}$. For a similar reason as in the proof of Lemma 3.9, we consider the intersection $S^{\prime \prime}=\left(\left(\mathbb{R}^{l} \times \mathbb{C}^{n}\right)\right.$ $0) \cap\left(\left(\mathbb{R}^{+}\right)^{l} \times\left\{z \in \mathbb{C}^{n}: f_{i}(z) \geq 0, i=1, \ldots\right\}\right)$, and we prove that $S^{\prime \prime} / T_{1}$ is connected and simply connected. We perturb the set $S^{\prime \prime}$ such that it has the same topology as a union of the sets $A_{i^{\prime}, i}=\left\{\left(a_{1}, \ldots, a_{l}\right) \in \mathbb{R}^{l}, z \in \mathbb{C}^{n}: a_{i^{\prime}}>\right.$ $\left.0, a_{J^{\prime}} \geq 0, f_{i}>0, f_{J} \geq 0\right\}$ for some $i^{\prime}=1, \ldots, l$ or for some $i=1, \ldots, p$, where $J^{\prime}=\{1, \ldots, l\}-i^{\prime}$ or $J=\{1, \ldots, p\}-i$ (only one of $i^{\prime}$ and $i$ is nonempty, so $J^{\prime}=\{1, \ldots, l\}$ or $\left.J=\{1, \ldots, p\}\right)$. We argue as we did in the proof of Lemma 3.9 that each $A_{i^{\prime}, i} / T_{1}$ is connected and simply connected, and the intersection of each two of these sets is connected. We glue them together and we use the Van-Kampen theorem to prove that $S^{\prime \prime} / T_{1}$ is connected and simply connected.

\section{Cross-section theorem and local normal form theorem}

In this section, we will first recall the Cross-section theorem due to Guillemin and Sternberg. These cross-sections will give us symplectic submanifolds with lower dimensional subgroup actions. Then, we will state the Local Normal Form Theorem for Hamiltonian Lie group actions, due to GuilleminSternberg, and Marle.

\subsection{Cross-section Theorem.}

Definition 4.3. Suppose that a group $G$ acts on a manifold $M$. Given a point $m$ in $M$ with isotropy group $G_{m}$, a submanifold $U \subset M$ containing $m$ is a slice at $\mathbf{m}$ if $U$ is $G_{m}$-invariant, $G \cdot U$ is a neighborhood of $m$, 
and the map

$$
G \times{ }_{G_{m}} U \longrightarrow G \cdot U, \quad[a, u] \longmapsto a \cdot u \text { is an isomorphism. }
$$

For instance, consider the co-adjoint action of $G=S U(2)$ or $S O(3)$ on $\mathbb{R}^{3}=\operatorname{Lie}(G)$. Recall that all the co-adjoint orbits through non-zero points in $\mathbb{R}^{3}$ are diffeomorphic to $S^{2}$ (these are generic co-adjoint orbits), and the co-adjoint orbit through 0 is 0 (this is a singular co-adjoint orbit). For $x \in \mathbb{R}^{3}, x \neq 0$, there is a unique ray $I_{x}$ passing through 0 and $x$. It is easy to see that the open ray $I_{x}^{\circ}=I_{x}-0$ is a slice at $x$. If $x=0$, then a slice at 0 is $\mathbb{R}^{3}$.

More generally, let us consider the co-adjoint action of a connected compact Lie group $G$ on $\mathfrak{g}^{*}$. For $x \in \mathfrak{g}^{*}$, let $U_{x}$ be the natural slice at $x$ for the co-adjoint action. Fix a (closed) positive Weyl chamber $\mathfrak{t}_{+}^{*}$, without loss of generality, we assume $x \in \mathfrak{t}_{+}^{*}$. Let $\tau \subset \mathfrak{t}_{+}^{*}$ be the open face of $\mathfrak{t}_{+}^{*}$ containing $x$ and let $G_{x}$ be the isotropy group of $x$ (all the points on $\tau$ have the same isotropy group). Then $U_{x}=G_{x} \cdot\left\{y \in \mathfrak{t}_{+}^{*} \mid G_{y} \subset G_{x}\right\}=G_{x} \cdot \bigcup_{\tau \subset \overline{\tau^{\prime}}} \tau^{\prime}$, and it is an open subset of $\mathfrak{g}_{\tau}^{*}=\mathfrak{g}_{x}^{*}$.

We have the following Cross-section theorem due to Guillemin and Sternberg (Theorem 26.7 in [7]; for the following version, see Corollary 2.3.6 in $[5])$.

Theorem 4.10. (Cross-section). Let $(M, \omega)$ be a symplectic manifold with a moment map $\phi: M \rightarrow \mathfrak{g}^{*}$ arising from an action of a compact connected Lie group $G$. Let $x$ be a point in $\mathfrak{g}^{*}$ and let $U$ be the natural slice at $x$. Then the cross-section $R=\phi^{-1}(U)$ is a $G_{x}$-invariant symplectic submanifold of $M$, where $G_{x}$ is the isotropy group of $x$. Furthermore, the restriction $\left.\phi\right|_{R}$ is a moment map for the action of $G_{x}$ on $R$.

4.2. Local normal form theorem. The following local normal form theorem describes up to equivariant isomorphism a neighborhood of an isotropic orbit in a Hamiltonian $G$-manifold.

Theorem 4.11. (Local normal form) $([\mathbf{8}])$ Let $(M, \omega)$ be a symplectic manifold with a Hamiltonian connected compact Lie group $G$ action. Assume $p \in M$, and the orbit $G \cdot p$ is isotropic. Let $H$ be the isotropy subgroup of $p$. Then a neighborhood of the orbit through $p$ in $M$ is equivariantly symplectomorphic to $G \times{ }_{H}\left(\mathfrak{b}^{\circ} \times V\right)$, where $\mathfrak{b}^{\circ}$ is the annihilator of $\mathfrak{b}=\operatorname{Lie}(H)$ in $\mathfrak{g}^{*}$ on which $H$ acts by the co-adjoint action, and $V$ is a complex vector space on which $H$ acts linearly and symplectically.

The equivalence relation on $G \times \times_{H}\left(\mathfrak{b}^{\circ} \times V\right)$ is given by $(g, a, v) \approx$ $\left(g h^{-1}, h \cdot a, h \cdot v\right)$ for $h \in H$.

The $G$ action on this local model is $g_{1} \cdot[g, a, v]=\left[g_{1} g, a, v\right]$, and the moment map on this local model is $\phi([g, a, v])=A d^{*}(g)(\phi(p)+a+\psi(v))$, where $\psi(v)$ is the moment map for the $H$ action on $V$. 


\section{Proof of Theorem 1.3 in the case of $G=S U$ (2) or $G=S O(3)$}

As an example, we will prove Theorem 1.3 for the action of $G=S U(2)$ and $G=S O(3)$. This will give us the flavor of the proof of Theorem 1.3.

If 0 is the only value in the moment map image, then by the definition of the moment map, $G$ acts trivially on $M$. So the theorem is trivial in this case.

Let us now assume that $\phi$ has non-zero values. Using Theorem 4.10, we will first reduce the proof of the theorem at non-zero values to a circle action case.

Lemma 5.11. Let $(M, \omega)$ be a connected, compact symplectic manifold equipped with a Hamiltonian $S U(2)$ or $S O(3)$ action. Assume the moment map image has non-zero values. Then, as fundamental groups of topological spaces, $\pi_{1}(M) \cong \pi_{1}\left(M_{\text {red }}\right)$, where $M_{\text {red }}$ is the symplectic quotient at any non-zero value of the moment map $\phi$.

Proof. Let $x \in i m(\phi)$, and $x \neq 0$. Let $I_{x}^{\circ}$ be the natural slice at $x$, and take $R=\phi^{-1}\left(I_{x}^{\circ}\right)$. By Theorem 4.10, $R$ is a symplectic submanifold with a Hamiltonian $S^{1}$ action whose moment map is $\left.\phi\right|_{R}$ and $\left.\phi\right|_{R}$ is proper onto its image. From Theorem 1.5, we can deduce that $R$ is connected. By Lemma 3.6 and Lemma 3.7 (see Remark 3.4), $\pi_{1}\left(M_{a}\right) \cong \pi_{1}\left(M_{b}\right)$ for any $a, b \in I_{x}^{\circ}$.

This same $S^{1}$ acts on $M$ with moment map being the projection of $\phi$ to $\mathbb{R}=\operatorname{Lie}\left(S^{1}\right)$. Let us use $\phi_{p}$ to denote this "projected" moment map. Suppose it has maximal value $z$. Then $z \in I_{x}^{\circ}$, and $\phi_{p}^{-1}(z)=\left.\phi\right|_{R} ^{-1}(z)$ is the maximum on $M$ of $\phi_{p}$. By Theorem 1.1, $\pi_{1}(M) \cong \pi_{1}\left(\phi_{p}^{-1}(z)\right) \cong$ $\pi_{1}\left(\phi_{p}^{-1}(z) / S^{1}\right) \cong \pi_{1}\left(\left.\phi\right|_{R} ^{-1}(z) / S^{1}\right) \cong \pi_{1}\left(M_{z}\right)$.

Lemma 5.12. Let $(M, \omega)$ be a connected, compact symplectic manifold equipped with a Hamiltonian $S U(2)$ or $S O(3)$ action. Assume 0 and $\epsilon$ small are in the moment map image. Then $\pi_{1}\left(M_{0}\right) \cong \pi_{1}\left(M_{G \cdot \epsilon}\right)$.

The proof of Lemma 5.12 relies on Theorem 3.8 and Lemma 5.13 below.

Lemma 5.13. Under the assumptions of Lemma 5.12, there exists a small $G$-invariant open neighborhood $U=\left\{x \in \mathbb{R}^{3}|| x \mid<\epsilon_{0}\right\}$, such that $U-0$ consists of regular values, and such that $\pi_{1}\left(\phi^{-1}(U) / G\right) \cong \pi_{1}\left(\phi^{-1}(U) / G-M_{0}\right)$.

Assume we have this. The proof of Lemma 5.12 goes as the following.

Proof. Assume that we have taken $U$ small enough such that we can use Theorem 3.8. Therefore, $\pi_{1}\left(M_{0}\right) \cong \pi_{1}\left(\phi^{-1}(U) / G\right)$. By Lemma 5.13, $\pi_{1}\left(\phi^{-1}(U) / G\right) \cong \pi_{1}\left(\phi^{-1}(U) / G-M_{0}\right)$. The space $\phi^{-1}(U) / G-M_{0}$ is 
homotopy equivalent to $\phi^{-1}(G \cdot \epsilon) / G=M_{\epsilon}$. To see this, by the Symplectic Cross-section Theorem, $\phi^{-1}(U)-\phi^{-1}(0)$ is the total space of a fibration over $S^{2}$ (a co-adjoint orbit) with fiber $\phi^{-1}(I)$, where $I$ is an open interval. Since all values in $I$ are regular, $\phi^{-1}(I)$ is isomorphic to $\phi^{-1}(\epsilon) \times I$ which is equivariantly homotopy equivalent to $\phi^{-1}(\epsilon)$. Therefore, $\phi^{-1}(U)-$ $\phi^{-1}(0)$ is equivariantly homotopy equivalent to the total space of a fibration over $S^{2}$ with fiber $\phi^{-1}(\epsilon)$, and this space is $\phi^{-1}(G \cdot \epsilon)$. So $\pi_{1}\left(M_{0}\right) \cong$ $\pi_{1}\left(M_{\epsilon}\right)$.

It remains to prove Lemma 5.13.

Proof. By equivariance of the moment map and by continuity, we see that if $\phi$ takes value 0 and a non-zero value, it has to take values in an open neighborhood of 0 . Since $M$ is compact, by considering a subcircle action, we see that if we take $U$ small enough, then $U-0$ consists of regular values of $\phi$.

By Lemma 2.1, $\phi^{-1}(U) / G$ is a stratified space. By $[\mathbf{1 3}], \phi^{-1}(0)$ and $M_{0}$ are stratified spaces. If there is only one stratum, then these spaces are manifolds. The sub-groups of $G=S U(2)$ or $S O(3)$ are finite, 1-dimensional, including $S^{1}$ and its normalizer $N\left(S^{1}\right)$, and $G$ itself. So $\phi^{-1}(0)$ and $M_{0}$ may contain strata with some or all of these orbit types.

We will remove strata of $M_{0}$ from $\phi^{-1}(U) / G$ in the order of lower dimensional ones (with "bigger" isotropy groups) to higher dimensional ones. By Lemma 2.3, we only need to prove that the link of each removed stratum is connected and simply connected.

Assume there is an $(H)$-stratum in $M_{0}$. By Theorem 4.11, a neighborhood $A$ in $M$ of a connected component of the $(H)$-stratum of $\phi^{-1}(0)$ is isomorphic to $A=G \times_{H}\left(\mathfrak{b}^{\perp} \times V\right)$, where $\mathfrak{b}^{\perp}$ is the annihilator of $\mathfrak{b}=\operatorname{Lie}(H)$ in $\mathfrak{g}^{*}$. Split $V=W \oplus V^{H}$. By Lemma 2.1, the link of the connected component of the $(H)$-stratum in $A / G$ is $L_{H}=S\left(\mathfrak{b}^{\perp} \times W\right) / H$.

1. Assume $H=G$. Then the above $L_{H}=L_{G}=S(W) / G$. Since the moment map image intersects a neighborhood of $0, W$ has to be a non-trivial complex $G$-representation, so $S(W)$ (with high dimension) is connected and simply connected. By Lemma 2.2 or by Theorem 2.7, $L_{G}$ is connected and simply connected.

2. Assume $H=N\left(S^{1}\right)$. Then the above $L_{H}=S\left(\mathbb{R}^{2} \times W\right) / N\left(S^{1}\right)$, where $\mathbb{R}^{2}$ is such that $\mathbb{R}^{2} \oplus \operatorname{Lie}^{*}\left(N\left(S^{1}\right)\right)=\mathfrak{g}^{*}$. In this case, $N\left(S^{1}\right)$ acts on $\mathbb{R}^{2}$ as the action of $O(2)$. If $W=0, L_{H}$ is a point. Otherwise, by Theorem 2.7, $L_{H}$ is connected and simply connected.

3. Assume $H=S^{1}$. Similar to 2 .

4. Assume $H=\Gamma$, where $\Gamma$ is a finite subgroup of $G$. Then the above link is $L_{H}=L_{\Gamma}=S\left(\mathbb{R}^{3} \times W\right) / \Gamma$. Since each element of $\Gamma$ belongs to a maximal torus of $G$, it has a non-zero fixed vector in $S\left(\mathbb{R}^{3}\right)$. By Theorem 2.7, $L_{\Gamma}$ is connected and simply connected. 


\section{Proof of Theorem 1.3}

In this section, we prove Theorem 1.3.

Let $G$ be a connected compact non-abelian Lie group with Lie algebra $\mathfrak{g}$ and dual Lie algebra $\mathfrak{g}^{*}$. Let $\mathfrak{t}_{+}^{*}$ be a fixed closed positive Weyl chamber of $\mathfrak{g}^{*}$. The set $\mathfrak{t}_{+}^{*}$ intersects each co-adjoint orbit at a unique point, and it consists of open faces with different dimensions. All the points on a fixed open face of $\mathfrak{t}_{+}^{*}$ have the same stabilizer group under the co-adjoint action. We advise the reader to distinguish Weyl chamber and the faces of the Weyl chamber in this section with the chamber and faces of the abelian moment polytope in Section 3 for which we used and we will use black letters.

By Theorem 1.5, $\phi(M) \cap \mathfrak{t}_{+}^{*}=\triangle^{\prime}$ is a convex polytope. Let us call the highest dimensional face $\tau^{P}$ of $\mathfrak{t}_{+}^{*}$ which contains values of $\phi$ the principal face, and let us call the generic values of $\phi$ on $G \cdot \tau^{P}$ generic values of $\phi$. Let $G_{\tau^{P}}$ (connected) be the stabilizer group of $\tau^{P}$ under the co-adjoint action. Let $U_{\tau^{P}}$ be the slice at $\tau^{P}$. By Theorem 4.10, $G_{\tau^{P}}$ acts on the principal cross-section $\phi^{-1}\left(U_{\tau^{P}}\right)$. Split $G_{\tau^{P}}=G_{\tau^{P}}^{\prime} \times T_{\tau^{P}}$, where $G_{\tau^{P}}^{\prime}$ is semi-simple, and $T_{\tau^{P}}$ is abelian. By Theorem 3.1 in [11] (see the cited theorem below), the semi-simple part $G_{\tau^{P}}^{\prime}$ acts trivially, only the connected central torus $T_{\tau^{P}}$ of $G_{\tau^{P}}$ acts on $\phi^{-1}\left(U_{\tau^{P}}\right)$ non-trivially. If $\tau^{P}$ is in the open positive Weyl chamber $\sigma$ of $\mathfrak{t}_{+}^{*}$, then $\phi^{-1}\left(U_{\sigma}\right)$ has the maximal torus $T$ action. Otherwise, the central torus $T_{\tau^{P}}$ of $G_{\tau^{P}}$ which acts on $\phi^{-1}\left(U_{\tau^{P}}\right)$ has a smaller dimension than the dimension of the maximal torus $T$ of $G$.

Let us write Theorem 3.1 in [11] in the following

Theorem 6.12. Let $G$ be a compact connected Lie group, and $M$ a connected Hamiltonian $G$-manifold with moment map $\phi: M \rightarrow \mathfrak{g}^{*}$.

a. There exists a unique open face $\tau^{P}$ of the Weyl chamber $\mathfrak{t}_{+}^{*}$ with the property that $\phi(M) \cap \tau^{P}$ is dense in $\phi(M) \cap \mathfrak{t}_{+}^{*}$.

b. The preimage $Y=\phi^{-1}\left(\tau^{P}\right)$ is a connected symplectic $T_{\tau^{P} \text {-invariant }}$ submanifold of $M$, and the restriction $\left.\phi\right|_{Y}$ of $\phi$ to $Y$ is a moment map for the action of $T_{\tau^{P}}$.

c. The set $G \cdot Y=\{g \cdot m \mid g \in G, m \in Y\}$ is dense in $M$.

Remark 6.8. For Theorem 1.3, without loss of generality, let us assume that the principal stabilizer type of the points in $M$ intersects the center of $G$ trivially. If not, the above intersection subgroup (which is contained in the center) is contained in all the stabilizer groups of the points in $M$, so we can divide it out and consider the quotient group action.

The proof of Theorem 1.3 consists of Lemmas 6.14-6.16.

Lemma 6.14. Let $(M, \omega)$ be a connected, compact symplectic manifold equipped with a Hamiltonian $G$ action with moment map $\phi$, where $G$ is 
a connected compact non-abelian Lie group. Assume that a G-invariant metric is chosen on $\mathfrak{g}^{*}$. Let $b \in \mathfrak{t}_{+}^{*}$ be the furthest moment map value from the origin. Then $\pi_{1}(M) \cong \pi_{1}\left(M_{b}\right)$.

Proof. Let the Weyl group act on $\triangle^{\prime}$. The image of $\triangle^{\prime}$ under this action is a polyhedron set (not necessarily convex) in $\mathfrak{t}^{*}$, and, we see that the Weyl group orbit through $b$ consists of the furthest points in $\mathfrak{t}^{*}$ to the origin, they are some boundary vertices of the polyhedron.

The maximal torus $T$ of $G$ acts on $M$ with moment map $\phi_{T}$ being the projection to $\mathfrak{t}^{*}$ of the $G$ moment map $\phi$. By Theorem 1.4, this moment map image is a convex polytope. The point $b$ (and its Weyl group images) is a boundary vertex of the image of $\phi_{T}$. To see this, take the line segment $o b$, take the hyperplane in $\mathfrak{g}^{*}$ perpendicular to $o b$. Only the $G$ moment map images on this hyperplane will be projected to $b$. But, there cannot be points other than $b$ on this hyperplane which are in the $G$ moment map image. If there was, then this point is further than $b$ to the origin, and the intersection of $\mathfrak{t}_{+}^{*}$ and the co-adjoint orbit through this point would be a point on $\triangle^{\prime}$ which is further than $b$ to the origin. So we have proved that $\phi^{-1}(b)=\phi_{T}^{-1}(b)$ is a fixed point set component of the $T$ action on $M$. Therefore $\phi^{-1}(b)$ is a compact symplectic manifold with a trivial $T$ action.

Let $G_{b}$ be the stabilizer group of $b$ under the co-adjoint action. Then $G_{b}$ acts on $\phi^{-1}(b)$. The maximal torus of $G_{b}$ is also $T$. Since $T$ acts trivially on $\phi^{-1}(b), G_{b}$ acts trivially on $\phi^{-1}(b)$. So $\pi_{1}\left(M_{b}\right) \cong \pi_{1}\left(\phi^{-1}(b)\right) \cong \pi_{1}(M)$ by Lemma 3.5.

Lemma 6.15. Let $(M, \omega)$ be a connected, compact symplectic manifold equipped with a Hamiltonian $G$ action with moment map $\phi$, where $G$ is a connected compact non-abelian Lie group. Let $\tau^{P} \subset \mathfrak{t}_{+}^{*}$ be the principal face, and let $a, b \in \tau^{P}$ be any two moment map values. Then $\pi_{1}\left(M_{a}\right) \cong \pi_{1}\left(M_{b}\right)$.

Proof. By Theorem 6.12, the principal cross-section $\phi^{-1}\left(U_{\tau^{P}}\right)=Y$ is a connected symplectic submanifold of $M$ with a torus $T_{\tau^{P}}$ action whose moment map is $\left.\phi\right|_{Y}$. Since $\left.\phi\right|_{Y}$ is proper onto its image, we can use Theorem 3.8. So we still have Lemma 3.8 which implies Lemma 3.7. By Lemmas 3.6 and 3.7, we have $\pi_{1}\left(Y_{a}\right) \cong \pi_{1}\left(Y_{b}\right)$ for all $a, b \in \tau^{P}$. By definition of the reduced spaces, this is to say $\pi_{1}\left(M_{a}\right) \cong \pi_{1}\left(M_{b}\right)$.

Lemmas 6.14 and 6.15 immediately imply the following special case of Theorem 1.3.

Corollary 6.1. Let $(M, \omega)$ be a connected, compact symplectic manifold equipped with a Hamiltonian $G$ action with moment map $\phi$, where $G$ is a connected compact non-abelian Lie group. Assume that the polytope $\phi(M) \cap$ $\mathfrak{t}_{+}^{*}=\triangle^{\prime}$ only lies on one face of the positive Weyl chamber, i.e., the moment 
polytope only has a principal face. Then, as fundamental groups of topological spaces, $\pi_{1}(M) \cong \pi_{1}\left(M_{\text {red }}\right)$, where $M_{\text {red }}$ is the symplectic quotient at any co-adjoint orbit of the moment map $\phi$.

For general cases, if we have Lemmas 6.14 and 6.15 , we are only left to show the following.

Lemma 6.16. Let $(M, \omega)$ be a connected compact symplectic manifold equipped with a Hamiltonian $G$ action with moment map $\phi$, where $G$ is a connected compact non-abelian Lie group. Let $\tau^{P}$ be the principal face of the moment polytope. Let $c$ be a value of $\phi$ which is not on $\tau^{P}$, i.e., $c$ is on a lower dimensional face of $\mathfrak{t}_{+}^{*}$. Let a be a generic value on $\tau^{P}$ very near c. Then $\pi_{1}\left(M_{c}\right) \cong \pi_{1}\left(M_{a}\right)$.

Now, assume $c \in \tau$, where $\tau$ is a face of $\mathfrak{t}_{+}^{*}$. Let $G_{\tau}$ be the stabilizer group of points on $\tau$ under the co-adjoint action. Then $G_{\tau}$ is a compact and connected Lie subgroup. Clearly, $G_{\tau}$ contains $T$. Let $U_{c}$ be the natural slice (see Section 4) at $c$. By Theorem 4.10, $U_{c}$ contains $\tau^{P}$. Let $R=\phi^{-1}\left(U_{c}\right)$ be the cross-section (see Theorem 4.10) on which $G_{\tau}$ acts with moment map being $\left.\phi\right|_{R}$. Since $G_{\tau} \cdot \phi^{-1}\left(\tau^{P}\right)$ is open, dense and connected in $R$ by Theorem $6.12, R$ is connected. By definition of the reduced spaces at $c$ and at $a$, to compare $\pi_{1}$ of the two quotients $M_{a}$ and $M_{c}$, we may restrict attention to $\left(R,\left.\omega\right|_{R}, G_{\tau},\left.\phi\right|_{R}\right)$. Split $G_{\tau}=G_{1} \times T_{2}$, where $G_{1}$ is semi-simple, and $T_{2}$ is abelian and it is the connected component of the center of $G_{\tau}$. The linear space spanned by the face $\tau$ is the dual Lie algebra of $T_{2}$. Since $\tau$ lies on the center of $\mathfrak{g}_{\tau}^{*}$, we may shift the moment map $\left.\phi\right|_{R}$ by $c$ such that the value $c$ corresponds to the value 0 of the shifted moment map $\left.\phi^{\prime}\right|_{R}$. So, without loss of generality, we assume that we have the Hamiltonian space $\left(R,\left.\omega\right|_{R}, G_{\tau},\left.\phi^{\prime}\right|_{R}\right)$, and we want to prove that $\pi_{1}\left(R_{0}\right) \cong \pi_{1}\left(R_{G_{\tau} \cdot a}\right)$, where $a \in \tau^{P}$ is a generic value near 0 in $\mathfrak{g}_{\tau}^{*}$. Although $R$ may not be compact, since $M$ is compact, the moment map $\left.\phi\right|_{R}$ is proper onto its image. So, if we take a small $G_{\tau}$-invariant neighborhood $U$ of 0 in $\mathfrak{g}_{\tau}^{*}$, we may assume that the $G$-equivariant gradient flow of $f=\|\phi\|^{2}$ restricts to the $G_{\tau}$-equivariant gradient flow of $\left\|\left.\phi\right|_{R}\right\|^{2}$ on $\left.\phi\right|_{R} ^{-1}(U)$. By Theorem 3.8, there exists a smaller $G_{\tau}$-invariant neighborhood $U^{\prime}$ of 0 , such that $\left.\phi\right|_{R} ^{-1}\left(U^{\prime}\right)$ is $G_{\tau}$-equivariantly homotopy equivalent to $\left.\phi\right|_{R} ^{-1}(0)$.

So we only need to prove the following general lemma.

Lemma 6.17. Let $(N, \omega)$ be a connected symplectic manifold equipped with a Hamiltonian $K$ action with moment map $\phi$ which is proper onto its image, where $K$ is a connected compact non-abelian Lie group. Assume that the moment map takes value at 0 and it takes values in a neighborhood of 0 in $\mathfrak{k}^{*}$ (not necessarily that the moment map image fills an open neighborhood of 
$0)$. Let a be a generic value near 0 in the positive Weyl chamber $\mathfrak{t}_{+}^{*}$. Then $\pi_{1}\left(N_{0}\right) \cong \pi_{1}\left(N_{a}\right)$.

Lemma 6.18. Under the assumptions of Lemma 6.17, assume in addition that $U \subset \mathfrak{k}^{*}$ is a small open invariant neighborhood of 0 such that $\phi^{-1}(U)$ equivariantly deformation retracts to $\phi^{-1}(0)$. Let $B$ be the set of values in $U$ which are on the faces other than the principal face $\tau^{P}$ of the closed positive Weyl chamber and the set of values which are on $\tau^{P}$ but not on the open connected chamber of generic values containing a. Then $\pi_{1}\left(\phi^{-1}(U) / K\right) \cong$ $\pi_{1}\left(\phi^{-1}(U) / K-\phi^{-1}(K \cdot B) / K\right)$.

Assume we have this lemma. Then the proof of Lemma 6.17 goes as the following.

Proof. Since $\phi^{-1}(U)$ equivariantly deformation retracts to $\phi^{-1}(0)$, we have $\pi_{1}\left(\phi^{-1}(U) / K\right) \cong \pi_{1}\left(N_{0}\right)$. By Lemma $6.18, \pi_{1}\left(\phi^{-1}(U) / K\right) \cong \pi_{1}\left(\phi^{-1}(U)\right.$ $\left./ K-\phi^{-1}(K \cdot B) / K\right) \cong \pi_{1}\left(N_{a}\right)$.

It is left to prove Lemma 6.18. The following two facts about the coadjoint action of a connected compact Lie group are needed in the proof of Lemma 6.18.

Proposition 6.2. Let $G$ be an n-dimensional connected compact Lie group with Lie algebra $\mathfrak{g}$ and dual Lie algebra $\mathfrak{g}^{*}$. Let $H \neq G$ be a subgroup of $G$ with Lie algebra Lie $(H)=\mathfrak{b}$, and let $\mathfrak{b}^{\circ}$ be the annihilator of $\mathfrak{b}$ in $\mathfrak{g}^{*}$. The space $\mathfrak{b}^{\circ}$ can be identified with the orthogonal complement $\mathfrak{b}^{\perp}$ of $\mathfrak{b}$ in $\mathfrak{g}$ for a suitable metric. The subgroup $H$ acts on $\mathfrak{b}^{\circ}$ by the co-adjoint action. Then, the smallest normal subgroup $N_{H}$ of $H$ containing the identity component of $H$ and all those elements of $H$ which have non-zero fixed points is $H$ itself.

Proof. (1) If $H$ is connected, we are done.

(2) Assume that $H$ is not connected. Let $H^{0}$ be the identity component of $H$. Then $H$ is generated by $H^{0}$ and finitely many elements, say $h_{1}, h_{2}, \ldots, h_{k}$. If we can prove that each $h_{i}, i=1, \ldots, k$ has a non-zero fixed point in $\mathfrak{b}^{\circ}$, then we are done. Let $T_{1}$ be a maximal torus of $H$. Then $T_{1} \subset H^{0}$. Let $T$ be a maximal torus of $G$ such that $T=T_{1} \times T_{2}$, where the dual Lie algebra $\mathfrak{t}_{2}$ of $T_{2}$ is contained in $\mathfrak{b}^{\circ}\left(T_{1}\right.$ or $T_{2}$ can be trivial). If $h_{i} \in T$, then $\mathfrak{t}_{2} \neq 0$. So $h_{i}$ fixes the subspace $\mathfrak{t}_{2}$ of $\mathfrak{b}^{\circ}$. If $h_{i} \notin T$, i.e., $h_{i}$ is in a different maximal torus $T^{\prime}$ other than $T$, then the dual Lie algebra $\mathfrak{t}^{\prime *}$ of $T^{\prime}$ has to have a non-zero component in $\mathfrak{b}^{\circ}$. Indeed, if $\mathfrak{t}^{\prime *} \subset \mathfrak{b}$, then $h_{i} \in T^{\prime} \subset H^{0}$, a contradiction. So $h_{i}$ fixes the above non-zero component in $\mathfrak{b}^{\circ}$.

Proposition 6.3. Let $G$ be a connected compact semi-simple non-abelian Lie group with Lie algebra $\mathfrak{g}$ and dual Lie algebra $\mathfrak{g}^{*}$. Let $H$ be a subgroup of $G$ with Lie algebra $\mathfrak{h}$. Let $\mathfrak{h}^{\circ}$ be the annihilator of $\mathfrak{h}$ in $\mathfrak{g}^{*}$. If $H \neq G$, then 
$H$ has at least codimension 2. If $H$ has codimension 2, i.e., if $\operatorname{dim}\left(\mathfrak{h}^{\circ}\right)=2$, then $H$ acts (co-adjoint action) on $S\left(\mathfrak{h}^{\circ}\right)=S^{1}$ transitively.

Proof. Let $\mathfrak{g}=\mathfrak{t} \oplus \bigoplus_{\alpha \in \mathcal{R}^{+}} M_{\alpha}$ be the (real) root space decomposition of $\mathfrak{g}$, where $\mathfrak{t}$ is the Lie algebra of the maximal torus $T_{G}$ of $G$, and $\mathcal{R}^{+}$is the set of positive roots. We know that each root space $M_{\alpha}$ is 2-dimensional, and for two generators $X, Y \in M_{\alpha}$, there is a $Z \in \mathfrak{t}$, such that $[Z, X]=2 X,[Z, Y]=$ $-2 Y,[X, Y]=2 Z$ (this corresponds to the Lie algebra of $S U(2)$ ). Let $T_{H}$ be the maximal torus of $H$. We can split $\mathfrak{h}$ similar to the splitting of $\mathfrak{g}$. If $\operatorname{dim}\left(T_{H}\right)<\operatorname{dim}\left(T_{G}\right)$, then at least one vector of $\mathfrak{t}$ is missing in $\operatorname{Lie}\left(T_{H}\right) \subset \mathfrak{h}$, and at least one $M_{\alpha}$ is missing in $\mathfrak{h}$. Indeed, let us assume that $\operatorname{dim}\left(T_{H}\right)=$ $\operatorname{dim}\left(T_{G}\right)-1$. Let $Z$ be the non-zero vector in $\mathfrak{t}$ but not in $\operatorname{Lie}\left(T_{H}\right)$. It is clear that the linear space $M_{\alpha}$ which has the above mentioned property with $Z$ cannot be in $\mathfrak{h}$ since $\mathfrak{h}$ is a Lie algebra. If only one generator $X \in M_{\alpha}$ was in $\mathfrak{h}$, then $X$ would contribute to a vector in $\operatorname{Lie}\left(T_{H}\right)$ which contradicts to the fact that $\operatorname{dim}\left(T_{H}\right)=\operatorname{dim}\left(T_{G}\right)-1$. Therefore, in the case that $\operatorname{dim}\left(T_{H}\right)<$ $\operatorname{dim}\left(T_{G}\right), H$ has at least codimension 3 in $G$. If $\operatorname{dim}\left(T_{H}\right)=\operatorname{dim}\left(T_{G}\right)$, then $\mathfrak{t} \subset \mathfrak{h}$. Since $H \neq G$, at least one $M_{\alpha}$ is missing in $\mathfrak{h}$. So, in this case, $H$ has at least codimension 2 in $G$. When codimension of $H$ is $2, \mathfrak{h}^{\circ}=M_{\alpha}$ for some $\alpha$. So there is an $S^{1}$ (generated by the above $Z$ ) in the maximal torus of $G$ which acts on $S\left(\mathfrak{h}^{\circ}\right)=S^{1}$ transitively.

Let us now proceed to prove Lemma 6.18. For the same reason as we made in Remark 3.5, we will prove Lemma 6.18 by induction on removing $\phi^{-1}(K \cdot \tau) / K$ in the order of lower dimensional faces $\left(\tau \mathrm{s}^{\prime}\right.$ whose preimage may have bigger stabilizer groups) to higher dimensional faces of the closed positive Weyl chamber. Let $C$ be the central face of the closed positive Weyl chamber $\mathfrak{t}_{+}^{*}$. Write $K=K_{1} \times T_{1}$, where $K_{1}$ is semi-simple, and $T_{1}$ is abelian (both $K_{1}$ and $T_{1}$ are connected). If $C$ is the principal face, then by Theorem 6.12, only the central torus $T_{1}$ acts on $N$. Corollary 6.1 addressed this case. In the following, we assume that $C$ is not the only face which contains the image of $\phi$. Let us first remove $\phi^{-1}(C) / K$ from $\phi^{-1}(U) / K$.

Lemma 6.19. Under the assumptions of Lemma 6.18, let $C$ be the central face of $\mathfrak{t}_{+}^{*}$. Assume that $C$ is not the only face which contains the image of $\phi$. Then $\pi_{1}\left(\phi^{-1}(U) / K\right) \cong \pi_{1}\left(\phi^{-1}(U) / K-\phi^{-1}(C) / K\right)$.

Proof. Let $K=K_{1} \times T_{1}$, where $K_{1}$ is semi-simple, and $T_{1}$ is abelian. Both $K_{1}$ and $T_{1}$ are connected by assumption. Let the Lie algebra of $K_{1}$ be $\mathfrak{k}_{1}$, and let its dual Lie algebra be $\mathfrak{k}_{1}^{*}$. Let the Lie algebra of $T_{1}$ be $\mathfrak{t}_{1}$, and let its dual Lie algebra be $\mathfrak{t}_{1}^{*}$. Then $C=\mathfrak{t}_{1}^{*}$.

We will remove strata of $\phi^{-1}(C) / K$ in the order of lower dimensional ones to higher dimensional ones and use Lemma 2.3 repeatedly. 
Now, assume there is a stratum with isotropy type $(H)$ (subgroups conjugate to $H$ ) in $\phi^{-1}(C) / K$. Then $H$ has the form of a product $H=H_{1} \times T^{\prime}$, where $H_{1}$ is a subgroup of $K_{1}$, and $T^{\prime}$ is a subgroup of $T_{1}$. Let $\mathfrak{h}_{1}^{\perp}$ be the annihilator of the Lie algebra $\mathfrak{h}_{1}=\operatorname{Lie}\left(H_{1}\right)$ in $\mathfrak{k}_{1}^{*}$, and let $\left(\mathfrak{t}^{\prime}\right)^{\perp}$ be the annihilator of the Lie algebra $\mathfrak{t}^{\prime}=\operatorname{Lie}\left(T^{\prime}\right)$ in $\mathfrak{t}_{1}^{*}$. Since every orbit in $\phi^{-1}(C)$ is isotropic, we can use Theorem 4.11. By Theorem 4.11, a neighborhood in $N$ of an orbit in $\phi^{-1}(C)$ with isotropy type $(H)$ is isomorphic to $A=K \times_{H}\left(\mathfrak{b}^{\perp} \times V\right)$, where $\mathfrak{b}^{\perp}$ is the annihilator of $\mathfrak{b}=\operatorname{Lie}(H)$ in $\mathfrak{k}^{*}=\operatorname{Lie}^{*}(K)$. Let $V=W \times V^{H}$. Write $A=K \times_{H}\left(\mathfrak{h}_{1}^{\perp} \times\left(\mathfrak{t}^{\prime}\right)^{\perp} \times\left(W \times V^{H}\right)\right)$. The quotient $A / K$ is $\left(\mathfrak{h}_{1}^{\perp} \times W\right) / H \times\left(\mathfrak{t}^{\prime}\right)^{\perp} \times V^{H}$. The $(H)$-stratum of $\phi^{-1}(C) / K$ in $A / K$ is $\left(\mathfrak{t}^{\prime}\right)^{\perp} \times V^{H}$. The link $L_{H}$ of this connected $(H)$ stratum is $S\left(\mathfrak{h}_{1}^{\perp} \times W\right) / H$. Now, we consider all the possible cases of $H$.

1. Assume that $H_{1}=K_{1}$. Then the above link is $S(W) /\left(K_{1} \times T^{\prime}\right)$. Since we assumed that the moment map image intersects not only the central face of the positive Weyl chamber, $W$ has to be a non-trivial complex $K_{1}$ representation. So $S(W)$ (with dimension at least 3 ) is connected and simply connected. Due to the fact that the quotient of $W$ by a finite abelian group $\Gamma$ is homeomorphic to $W$, we may assume that $T^{\prime}$ is connected. By Lemma 2.2, the link $L_{H}$ is connected and simply connected.

2. Assume $H_{1} \neq K_{1}$. By Proposition $6.3, \operatorname{dim}\left(\mathfrak{h}_{1}^{\perp}\right) \geq 2$.

First, let us assume that $W \neq 0$, then $\operatorname{dim}\left(\mathfrak{h}_{1}^{\perp} \times W\right)>2$. So $S\left(\mathfrak{h}_{1}^{\perp} \times W\right)$ is connected and simply connected. By Proposition 6.2 and Theorem $2.7\left(T^{\prime}\right.$ acts on $\mathfrak{h}_{1}^{\perp}$ trivially), the link $S\left(\mathfrak{h}_{1}^{\perp} \times W\right) /\left(H_{1} \times T^{\prime}\right)$ is connected and simply connected.

Next, let us assume that $\operatorname{dim}\left(\mathfrak{h}_{1}^{\perp}\right)=2$ and $W=0$. The link is $S\left(\mathfrak{h}_{1}^{\perp}\right) / H$. By Proposition 6.3, this link is a point, therefore connected and simply connected.

Now, let us assume that $\mathfrak{h}_{1}^{\perp}>2$ and $W=0$. By Proposition 6.2 and Theorem 2.7, the link is connected and simply connected.

Proof of Lemma 6.18:

Proof. By assumption, $0 \in i m(\phi)$ and $i m(\phi)$ intersects a small neighborhood of 0 . If the image of $\phi$ only lies on the central face $C$ of the positive Weyl chamber, then the main theorem comes down to the case of Corollary 6.1. Now, we assume that the image of $\phi$ intersects not only one face of the positive Weyl chamber.

Lemma 6.19 removed $\phi^{-1}(C) / K$ from $\phi^{-1}(U) / K$.

Assume now that the image of $\phi$ intersects another higher dimensional face $\tau$ other than $\tau^{P}$ of the positive Weyl chamber. Suppose the stabilizer group of $\tau$ under the co-adjoint action is $K_{\tau}$. Let $U_{\tau}$ be the natural slice at $\tau$. Then by the symplectic cross-section theorem, $Y_{\tau}=\phi^{-1}\left(U_{\tau}\right)$ is a symplectic submanifold with a $K_{\tau}$ action. The face $\tau$ lies on the central 
dual Lie algebra of $K_{\tau}$. Similar to Lemma 6.19 , we remove $\phi^{-1}(\tau) / K_{\tau}$ from $\left(\phi^{-1}(U) \cap Y_{\tau}\right) / K_{\tau}$. In order to remove $\phi^{-1}(K \cdot \tau) / K$ from $\phi^{-1}(U) / K$, we only need to notice that, by equivariance of the moment map, each stratum of $\phi^{-1}(K \cdot \tau) / K$ has the same link in $\phi^{-1}(U) / K$ as the corresponding stratum of $\phi^{-1}(\tau) / K_{\tau}$ in $\left(\phi^{-1}(U) \cap Y_{\tau}\right) / K_{\tau}$. Indeed, to prove this, observe that $K \cdot Y_{\tau}$ is equivariantly diffeomorphic to the bundle $K \times_{K_{\tau}} Y_{\tau}$ over the co-adjoint orbit $K / K_{\tau}$. So each connected stratum $\tilde{S}$ in $K \cdot Y_{\tau}$ is $\tilde{S}=K \times_{K_{\tau}} \tilde{S}^{\prime}$, where $\tilde{S}^{\prime}$ is the corresponding stratum in $Y_{\tau}$. A neighborhood of $\tilde{S}$ in $K \cdot Y_{\tau}$ corresponds to a neighborhhood of $\tilde{S}^{\prime}$ in $Y_{\tau}$. Therefore, the quotient stratum $\tilde{S} / K$ in $K \cdot Y_{\tau} / K$ has the same link as $\tilde{S}^{\prime} / K_{\tau}$ in $Y_{\tau} / K_{\tau}$. If we restrict to the invariant set $\phi^{-1}(U)$, the same property still holds.

We can remove similarly $\phi^{-1}\left(K \cdot \tau^{\prime}\right) / K$ from $\phi^{-1}(U) / K$ if the image of $\phi$ intersects other non-principal faces $\tau^{\prime}$ 's' of the positive Weyl chamber.

Now assume only $\phi^{-1}\left(K \cdot\left(\tau^{P} \cap U\right)\right) / K$ is remaining. If all the values on $\tau^{P} \cap U$ are regular, we are done. Otherwise, we will remove and "flow" in the following way until only the connected open chamber containing $a$ is remaining. After we "removed" all the non-principal faces of the positive Weyl chamber, if on the "verge" of $\tau^{P} \cap U$, there is an open connected chamber $U^{\prime}$ not containing $a$, we use the gradient flow of suitable components of the $T$ moment map to deformation retract $\phi^{-1}\left(U^{\prime}\right)$ to $\phi^{-1}(\mathcal{F} s)$, where $\mathcal{F} s$ are certain singular faces around $U^{\prime}$. Correspondingly, by equivariance of $\phi$ again, $\phi^{-1}\left(K \cdot U^{\prime}\right)$ deformation retracts to $\phi^{-1}(K \cdot \mathcal{F} s)$. Then, we use Lemma 3.8 to remove $\phi^{-1}(\mathcal{F} s) / T_{\tau^{P}}$ from the remaining part of $\phi^{-1}\left(\tau^{P} \cap U\right) / T_{\tau^{P}}$, and we use equivariance as above to remove $\phi^{-1}(K \cdot \mathcal{F} s) / K$. Or, we may only need to do removing if there is only one connected open chamber which is the one containing $a$ is left in $\tau^{P} \cap U$ (the other singular faces are in the closure of this chamber). We may need to repeat the procedure until only the connected open chamber containing $a$ remains.

Now, we have removed all $\phi^{-1}(K \cdot B) / K$ from $\phi^{-1}(U) / K$. The remaining space is homotopy equivalent to $\phi^{-1}(K \cdot a) / K=N_{K} \cdot a$, where $a$ is a generic value in the chamber containing $a$.

\section{Proof of Theorem 1.6}

In this section, we will prove Theorem 1.6. For simply connected manifold $M$, Armstrong's theorem (Theorem 2.7) tells us that $\pi_{1}(M) \cong \pi_{1}(M / G)$. In this case, by Theorems 1.1-1.3, we have Theorem 1.6. In this section, we use our method of removing to give a direct proof of Theorem 1.6. Since we proved that all reduced spaces have isomorphic fundamental groups, we only need to prove that $M / G$ has the same fundamental group as that of a particular reduced space. 
In Lemma 3.8, we did removing from "one side", i.e., we took $\overline{U^{\prime}}$. Now, let us do a removing from "all sides", i.e., we take $U$ itself. The proof is even simpler.

Lemma 7.20. Assume we have the assumptions of Theorem 1.6, where $G$ is a torus $T$. Let $\mathcal{F}$ be a face of the moment polytope $\triangle$ which is not a chamber. Let $U$ be a small open neighborhood of $\mathcal{F}$ on $\triangle(U$ does not intersect the faces which are in the closure of $\mathcal{F})$. Let $S$ be the set of singular orbits (or non-generic orbits) in $\phi^{-1}(\mathcal{F})$. Then $\pi_{1}\left(\phi^{-1}(U) / T\right) \cong$ $\pi_{1}\left(\phi^{-1}(U) / T-S / T\right)$.

Proof. Assume that the dimension of the face $\mathcal{F}$ is $m$ with $m \geq 0$. We do the removing from lower dimensional strata to higher dimensional strata. As we did before, we only need to check that the link of the removed stratum is connected and simply connected.

As in the proof of Lemma 3.8, we split the torus $T=T^{n-m} \times T^{m}$. The possible stabilizer types of the strata in $\phi^{-1}(\mathcal{F})$ have the form $H=\left(T_{1} \times\right.$ $\left.\Gamma^{\prime}\right) \times \Gamma$, where $T_{1}$ is a connected subgroup of $T^{n-m}, \Gamma^{\prime}$ is a finite subgroup of $T^{n-m}$, and $\Gamma$ is a finite subgroup of $T^{m}$.

By Theorem 3.9, a neighborhood in $M$ of an orbit with isotropy type $(H)$ is isomorphic to $A=T \times{ }_{H}\left(\mathbb{R}^{l} \times \mathbb{R}^{m} \times V\right)$. Split $V=W \times V^{H}$. If $W=0$, then all the points in $A$ have the same stabilizer group. Since $A$ is open, this stabilizer group is the generic stabilizer group. So we assume that $W \neq 0$ by our assumption. The $(H)$-stratum in $A$ which was mapped to $\mathcal{F}$ is $T \times{ }_{H}\left(\mathbb{R}^{m} \times V^{H}\right)$. The quotient of $A$ by $T$ is $A / T=\mathbb{R}^{l} \times \mathbb{R}^{m} \times V^{H} \times$ $W / H$. The link $L_{H}$ of the corresponding quotient $(H)$-stratum in $A / T$ is $S\left(\mathbb{R}^{l} \times W\right) / H$. The possible cases of $H$ (or of $l$ ) are:

1. In the case $l=0$, since the moment map value fills the neighborhood $U, W$ has to be a non-trivial representation of $H$ with a non-trivial moment map. So either $L_{H}$ is a point, in the case of $\operatorname{dim}(W)=$ 2 , therefore connected and simply connected, or, it is connected and simply connected by the fact that $W / \Gamma$ is homeomorphic to $W$ and by applying Lemma 2.2 .

2. In the case $l \neq 0, S\left(\mathbb{R}^{l} \times W\right.$ ) is simply connected ( $W$ is a non-trivial complex vector space). By Theorem $2.7, L_{H}$ is connected and simply connected.

Lemmas 3.8, 6.19, and 7.20 did "local removing", namely, we chose small neighborhoods of a value or of a face on the moment polytope. Note that the removing itself does not depend on the gradient flow. In Lemmas 3.8 and 6.19, we chose $U$ to be small neighborhoods of one value. As in Lemma 7.20, if only for the purpose of removing (not for the purpose of comparing $\pi_{1}$ of 
the reduced spaces at nearby values), we could have taken $U$ in Lemmas 3.8 and 6.19 to be an open small neighborhood of the face we are considering. Now, if we consider the global quotient $M / G$, we can do the same removing in $M / G$, since the quotient of an orbit is only "linked" to its neighborhood in the space $M / G$.

Another observation is that the gradient flow (or negative gradient flow) of the components of the moment map or of the moment map square always retracts less singular regions on the manifold to more singular ones.

Lemmas 3.8, 6.19, and 7.20 allow us to remove (the quotients of certain orbits). By using the gradient flow of suitable components of the moment map, we can retract regular regions to singular ones. These two operations are the main points of the proof of Theorem 1.6.

Proof of Theorem 1.6 for $S^{1}$ actions:

Proof. Assume that the moment map takes critical values at $a_{0}, a_{1}, \ldots, a_{n}$, and we have $a_{0}<a_{1}<\cdots<a_{n}$.

By Lemma 3.8 and an argument by the Van-Kampen theorem, we have $\pi_{1}\left(M / S^{1}\right) \cong \pi_{1}\left(M / S^{1}-M_{a_{n}}\right)$. The right hand side is equal to $\pi_{1}\left(\phi^{-1}\left(\left[a_{0}\right.\right.\right.$, $\left.\left.\left.a_{n}\right)\right) / S^{1}\right)$. Now the negative gradient flow of $\phi$ equivariantly deformation retracts $\phi^{-1}\left(\left[a_{0}, a_{n}\right)\right)$ to $\phi^{-1}\left(\left[a_{0}, a_{n-1}\right]\right)$. So $\pi_{1}\left(\phi^{-1}\left(\left[a_{0}, a_{n}\right)\right) / S^{1}\right) \cong$ $\pi_{1}\left(\phi^{-1}\left(\left[a_{0}, a_{n-1}\right]\right) / S^{1}\right)$. By Lemma 3.8 and a similar argument by the VanKampen theorem as the above, $\pi_{1}\left(\phi^{-1}\left(\left[a_{0}, a_{n-1}\right]\right) / S^{1}\right) \cong \pi_{1}\left(\phi^{-1}\left(\left[a_{0}, a_{n-1}\right)\right)\right.$ $\left./ S^{1}\right)$. We use the negative gradient flow of $\phi$ again to "flow down" the next regular region $\phi^{-1}\left(\left(a_{n-2}, a_{n-1}\right)\right)$ to $\phi^{-1}\left(\left[a_{0}, a_{n-2}\right]\right)$. We repeatedly use the above procedure of removing and "flowing" until we reach $\pi_{1}\left(M / S^{1}\right) \cong \pi_{1}\left(M_{a_{0}}\right)$.

Proof of Theorem 1.6 for $G=T$ actions:

Proof. Different removing and flowing process can achieve the proof. The moment polytope $\triangle$ consists of faces with different dimensions; and, it may have one or more than one connected open chambers. The main tools we can use, or the main points of the proof are: (a) We can use Lemma 7.20 to remove singular faces on the boundary of $\triangle$. (b) The inverse image of a singular face in the "interior" of $\triangle$ may contain regular orbits (orbits with generic stabilizer group). Whenever after we use Lemma 7.20 for such a singular face, the gradient flow of some components of the moment map always takes the remaining regular orbits to an appropriate regular region (the inverse image of an open chamber). (c) We can start to remove from a vertex on the boundary of $\triangle$. We follow the principle we made in Remark 3.5 about the order of removing. We deform when it is allowed by using the gradient flow of suitable components of the moment map. When we encounter a removing of a singular face from the closure of 
only one connected chamber, we may use Lemma 3.8. (d) We can choose different process. In the end, we arrive at $\pi_{1}(M / G) \cong \pi_{1}\left(M_{b}\right)$, where $b$ is a value on $\triangle$.

Proof of Theorem 1.6 for non-abelian $G$ actions:

Proof. We use the method of Lemma 6.19 to inductively remove $\phi^{-1}(G \cdot \tau) / G$ from $M / G$ for the faces $\tau \mathrm{s}^{\prime}$ which are not the principal face $\tau^{P}$ of the closed positive Weyl chamber (Again, we may need the cross-section theorem to do some removing in the cross-section, and then use equivariance of the moment map. For this, see the proof of Lemma 6.18. I would like to stress that, the removing itself does not depend on the gradient flow.). Assume, now, we have $\pi_{1}(M / G) \cong \pi_{1}\left(\phi^{-1}\left(G \cdot \tau^{P}\right) / G\right)$. By equivariance of the moment map, the two spaces $\phi^{-1}\left(G \cdot \tau^{P}\right) / G$ and $\phi^{-1}\left(\tau^{P}\right) / T$ are the same. We use the method for torus actions to prove that $\pi_{1}\left(\phi^{-1}\left(\tau^{P}\right) / T\right) \cong \pi_{1}\left(M_{b}\right)$ for some value $b \in \tau^{P}$.

\section{References}

[1] M.A. Armstrong, Calculating the fundamental group of an orbit space, Proc. Amer. Math. Soc. 84(2), (1982), 267-271.

[2] M. Atiyah, Convexity and commuting Hamiltonians, Bull. Lond. Math. Soc. 14 (1982), 1-15.

[3] G. Bredon, Introduction to compact transformation groups, Academic Press, New York, 1972.

[4] W. Chen, A homotopy theory of orbispaces, math. AT/0102020.

[5] V. Guillemin, E. Lerman, and S. Sternberg, Symplectic fibrations and multiplicity diagrams, Cambridge University Press, Cambridge, 1996.

[6] V. Guillemin and S. Sternberg, Convexity properties of the moment mapping, Invent. Math. 67(3), (1982), 491-513.

[7] V. Guillemin and S. Sternberg, Symplectic techniques in physics, Cambridge University Press, Cambridge, 1990.

[8] V. Guillemin and S. Sternberg, A normal form for the moment map, in 'Differential geometric methods in mathematical physics' (S. Sternberg, ed.), Reidel, Dordrecht, Holland, 1984.

[9] F.C. Kirwan, Convexity properties of the moment mapping III, Invent. Math. 77 (1984), 547-552.

[10] E. Lerman, Gradient flow of the norm squared of a moment map, math. SG/0410568.

[11] E. Lerman, E. Meinrenken, S. Tolman, and C. Woodward, Nonabelian convexity by symplectic cuts, Topology 37(2), (1998), 245-259.

[12] H. Li, $\pi_{1}$ of Hamiltonian $S^{1}$ manifolds, Proc. Amer. Math. Soc. 131(11), (2003), 3579-3582.

[13] R. Sjamaar and E. Lerman, Stratified symplectic spaces and reduction, Ann. Math. 134(2), (1991), 375-422. 
[14] Y. Takeuchi and M. Yokoyama, The geometric realizations of the decompositions of 3-orbifold fundamental groups, Topology Appl. 95(2), (1999), 129-153.

[15] C. Woodward, The Yang-Mills heat flow on the moduli space of framed bundles on a surface, math.SG/0211231.

Department of Mathematics,

UNIVERSITY OF LUXEMBOURG,

162A, Ave De la FAiencerie,

L-1511, LuXEMBOURG

E-mail address: li.hui@uni.lu

Received 10/5/2006, accepted 2/3/2007

This work was supported in part by the Center for mathematical analysis, geometry, and dynamical systems, IST, Lisbon, Portugal where I found the method of the proof; and it was supported in part by the research grant R1F105L15 of professor Martin Schlichenmaier in Luxembourg University where I wrote most part of the paper. I thank both institutes and the people there for support and hospitality. I would like to thank Sue Tolman for all her discussion, especially her explanation on symplectic cross section theorem; and I thank Gustavo Granja for his nice discussion on Topology when I was studying $S U(2)$ and $S O(3)$ actions. I thank Oleg Sheinman for some discussion on Lie groups and for his pointing out Armstrong's theorem. Finally, I thank the referee for his comments. 